\title{
New Constructions of Zero-Correlation Zone Sequences
}

\author{
Yen-Cheng Liu, Ching-Wei Chen, and Yu T. Su
}

\begin{abstract}
In this paper, we propose three classes of systematic approaches for constructing zero correlation zone (ZCZ) sequence families. In most cases, these approaches are capable of generating sequence families that achieve the upper bounds on the family size $(K)$ and the ZCZ width $(T)$ for a given sequence period $(N)$.

Our approaches can produce various binary and polyphase ZCZ families with desired parameters $(N, K, T)$ and alphabet size. They also provide additional tradeoffs amongst the above four system parameters and are less constrained by the alphabet size. Furthermore, the constructed families have nested-like property that can be either decomposed or combined to constitute smaller or larger ZCZ sequence sets. We make detailed comparisons with related works and present some extended properties. For each approach, we provide examples to numerically illustrate the proposed construction procedure.
\end{abstract}

\section{Index Terms}

Hadamard matrix, mutually orthogonal complementary set of sequences, periodic correlation, upsampling, zero-correlation zone (ZCZ) sequence.

Y.-C. Liu and Y. T. Su (correspondence addressee) are with the Institute of Communications Engineering, National Chiao Tung University, Hsinchu, Taiwan (email: ycliu@ieee.org; ytsu@nctu.edu.tw). C.-W. Chen is with National Instruments Taiwan Corp., Taipei, Taiwan (email: penguinjazzy@gmail.com). The material in this paper was presented in part at the IEEE 2009 International Symposium on Information Theory. 


\section{INTRODUCTION}

Families of sequences with some desired periodic or aperiodic autocorrelation (AC) and crosscorrelation (CC) properties are useful in communication and radar systems for applications in identification, synchronization, ranging, or/and interference mitigation. For example, to minimize the multiple access interference (MAI) and self-interference (e.g., inter-symbol interference) in a multi-user, multi-path environment or to avoid inter-antenna interference in a multipleinput, multiple-output system, one would like to have an ideal sequence set whose periodic AC functions are nonzero only at the zeroth correlation lag $(\tau=0)$ and whose pairwise periodic $\mathrm{CC}$ values are identically zero at any $\tau$ for all pairs of sequences. Similar aperiodic properties are called for in designing pulse compressed radar signal or two-dimensional array waveforms to have an impulse-like ambiguity function satisfying the resolution requirements.

Unfortunately, the ideal sequence set does not exist, i.e., it is impossible to have impulse-like $\mathrm{AC}$ functions and zero $\mathrm{CC}$ functions simultaneously in a sequence set. In fact, bounds on the magnitude of $\mathrm{CC}$ and $\mathrm{AC}$ values derived in [1] and [2] suggest that the design of sequence sets involves the tradeoff between $\mathrm{AC}$ and $\mathrm{CC}$ values. An alternate compromise is to require that the ideal $\mathrm{AC}$ and $\mathrm{CC}$ properties be maintained only at correlation lags within a window called zero-correlation zone (ZCZ) [3]. Sequences with such properties are known as ZCZ sequences. Little or no system performance degradation results if the correlation values outsides the ZCZ are immaterial to the application of concern. For example, if the maximum channel delay spread $T_{m}$ and the maximum distance between a base station and co-channel users $D_{m}$ are known, a direct sequence spread spectrum based multiple access system using a family of ZCZ sequences with ZCZ width $|\tau| \leq T_{m}+2 D_{m} / c$, where $c$ is the speed of light, will be able to suppress MAI and multipath self interference.

Other than the restrictions on the magnitude of correlation values, practical implementation concerns prefer that the choice of the sequence period be flexible and the family size be as large as possible while keeping the desired AC and CC properties intact. One also hope that the elements of the sequences be drawn from an alphabet set as small as possible.

Various ZCZ sequence generation methods have been proposed [4]-[20]. The methods pre- 
sented in [4]-[6] are based on complementary sequence sets. Interleaving techniques are shown to be effective in constructing ZCZ sequences [7]-[9]. They can be generalized to construct two-dimensional (2-D) ZCZ arrays [10], [11] as well. Sets of ZCZ sequences derived from manipulating perfect sequences were suggested in [12] and [13]. Park et al. [14] construct sequences that has nonzero AC only at subperiodic correlation lags and zero $\mathrm{CC}$ across all lags. By requiring the transform domain sequences to satisfy some special properties, [15], [16] present methods that generate ZCZ sequences having zero CC across all lags. Some ZCZ sequence sets can be partitioned into smaller subsets so that the zero-CC zone of any two sequences drawn from different subsets are wider than that among intra-subset sequences. Ternary or polyphase sequences with such a property have been constructed via interleaving techniques [17], [18] and in [19], [20] quadrature amplitude-modulated (QAM) sequences are shown to be derivable from binary or ternary sequences.

In this paper, we present three systematic approaches for generating families of sequences whose periodic $\mathrm{AC}$ and $\mathrm{CC}$ functions satisfy a variety of $\mathrm{ZCZ}$ requirements. While some known ZCZ sequence construction methods employing Hadamard matrices in time domain (e.g., [9], [13]), our first approach uses such matrices to meet the desired transform domain properties of a ZCZ sequence set instead. Sequence sets generated from this approach are, by construction, optimal in the sense that the upper bounds for family sizes and ZCZ widths are achieved. We further employ a filtering operation to convert sequences of nonconstant modulus symbols into polyphase ones without changing the correlation properties.

Based upon a basic binary sequence (to be defined in Section IV) whose AC function satisfies the ZCZ requirement, the second approach generates ZCZ sequence families by a special nonuniform upsampling on unitary matrices. The construction of basic sequences seems trivial and straightforward, but from these simple sequences we are able to synthesize desired polyphase ZCZ sequences through some refining steps that include nonuniform upsampling and filtering.

Our third approach invokes the notion of complementary set of sequences [21], [22]. It bears the flavor of the second approach and makes use of a basic binary sequence which meets the ZCZ constraint as well as a collection of mutually orthogonal complementary sets. While this 
method is capable of generating binary sequences with sequence parameters identical to those given in [4] and [6], it can also produce polyphase sequence sets that are unobtainable by the conventional complementary set-based approaches.

The rest of this paper is organized as follows. We introduce basic definitions and properties related to our investigation in the next section. Section III begins with a brief summary of important transform domain properties, followed by the analysis and synthesis of the proposed transform domain approach. We then show some ZCZ sequence sets generated by the transform domain method in subsection III-D. The direct synthesis method is presented in section IV and construction examples are given in subsection IV-E. In section V, a complementary sequence set based extension of the second approach is proposed, followed by numerical construction examples given in subsection V-D. For each proposed approach, we tabulate the parametric constraint comparisons with related methods. More detailed comparisons and discussions are given in the form of remarks. Finally, some concluding notes are provided in Section VI.

\section{Definitions And Fundamental Properties}

Definition 1: An $(N, K)$ sequence set $\mathrm{X}$ is a set of $K$ sequences of period $N$.

Definition 2: The periodic CC function of two period- $N$ sequences $u \equiv\{u(n)\}$ and $v \equiv$ $\{u(n)\}$ is defined as

$$
\theta_{u v}(\tau)=\sum_{n=0}^{N-1} u(n) v^{*}(n-\tau)=u(\tau) \circledast v^{*}(-\tau),
$$

where $\circledast$ denotes the circular convolution.

Thus, the periodic AC function of sequence $u$ is simply $\theta_{u u}(\tau)$. Since these $\mathrm{CC}$ and AC functions are also of period $N$, to simplify the discussion we shall, throughout this paper, limit the representations and examples of sequences or sequence sets to a single period $(0 \leq \tau \leq N-1)$ unless necessary.

Definition 3: A sequence $\{u(n)\}$ that has an impulse-like (or ideal) AC function, i.e., $\theta_{u u}(\tau)=$ 
$\theta_{u u}(0) \delta(\tau)$, is called a perfect sequence, where

$$
\delta(\tau) \stackrel{\text { def }}{=}\left\{\begin{array}{cc}
1, & \tau=0 \\
0, & \tau \neq 0
\end{array}\right.
$$

is the Dirac delta function.

Definition 4: A sequence $\left\{u_{v}(n)\right\}$ is said to be obtained from filtering the sequence $u=$ $\{u(n)\}$ by the sequence $v=\{v(n)\}$ of the same period if

$$
u_{v}(n) \stackrel{\text { def }}{=} u(n) \circ v(n) \stackrel{\text { def }}{=} u(n) \circledast v^{*}(-n) \equiv \theta_{u v}(n)
$$

Definition 5: An $(N, K)$ sequence set, $\mathbf{C}=\left\{C_{0}, C_{1}, \cdots, C_{K-1}\right\}$ is called an $(N, K, T) \mathrm{ZCZ}$ sequence family (or set) if $\forall C_{i}, C_{j} \in \mathbf{C}, i \neq j, \theta_{C_{i} C_{j}}(\tau)=0$ and $\theta_{C_{i} C_{i}}(\tau)=\theta_{C_{i} C_{i}}(0) \delta(\tau)$, $|\tau|_{N} \leq T<N$ where $T$ is the ZCZ width and $|k|_{N} \stackrel{\text { def }}{=} k \bmod N$.

In [23], it was proved that

Lemma 1: The sequence period $N$, cardinality $K$ and ZCZ width $T$ of an $(N, K, T) \mathrm{ZCZ}$ family must satisfy the inequality

$$
K(T+1) \leq N
$$

For \pm 1 -valued binary sequence set, the bound becomes more tight [7]

$$
K T \leq \frac{N}{2}, K>1
$$

This lemma describes the fundamental tradeoff among the sequence period, family size, and ZCZ width. For a fixed $N$, increasing the family size must be achieved at the cost of reduced ZCZ width and vice versa. Note that for a set with a single perfect sequence, (4) is automatically satisfied because $K=1$ and $T=N-1$.

Definition 6: An $N \times N$ matrix $\mathrm{U}$ is called a Hadamard matrix of order $N$ if and only if it satisfies two conditions:

(i) Unimodularity: the components of $\mathbf{U}$ are of the same magnitude $\sqrt{P}$; 
(ii) Orthogonality: $\mathbf{U U}^{H}=N P \mathbf{I}_{N}$ where $\mathbf{I}_{N}$ is the $N \times N$ identity matrix and $(\cdot)^{H}$ denotes the conjugate transpose of the enclosed matrix.

Definition 7: The Matrix

$$
\mathbf{F}_{M}=\left[\begin{array}{ccccc}
1 & 1 & 1 & \cdots & 1 \\
1 & W_{M}^{-1} & W_{M}^{-2} & \cdots & W_{M}^{-(M-1)} \\
1 & W_{M}^{-2} & W_{M}^{-4} & \cdots & W_{M}^{-2(M-1)} \\
\vdots & \vdots & \vdots & \ddots & \vdots \\
1 & W_{M}^{-(M-1)} & W_{M}^{-2(M-1)} & \cdots & W_{M}^{-(M-1)^{2}}
\end{array}\right]
$$

is called the $M$-discrete Fourier transform ( $M$-DFT) matrix, where $W_{M}^{k}=e^{j 2 \pi k / M}$, and its Hermitian $\mathbf{F}_{M}^{H}=\mathbf{F}_{M}^{-1}$ is called the inverse $M$-DFT ( $M$-IDFT) matrix. The set of complex $M$ th roots of unity, $\left\{W_{M}^{k}: k=0,1, \cdots, M-1\right\}$, is called the $M$-ary phase-shift keying (M-PSK) set and a sequence with elements from the $M$-PSK constellation is called an $M$-PSK sequence or a polyphase sequence in general.

Note that DFT matrices form a subcalss of the so-called Butson Hadamard matrices [24].

Definition 8: The $k$ th Kronecker power of matrix $\mathbf{U}$, denoted by $\otimes^{k} \mathbf{U}$, is defined as

$$
\otimes^{k} \mathbf{U}=\underbrace{\mathbf{U} \otimes \mathbf{U} \otimes \cdots \otimes \mathbf{U}}_{\mathbf{U} \text { appears } k \text { times }}
$$

where $\otimes$ denotes the Kronecker product.

Definition 9: The matrices

$$
\mathbf{H}_{2}=\left[\begin{array}{rr}
1 & 1 \\
1 & -1
\end{array}\right]
$$

and

$$
\mathbf{H}_{2^{n}}=\otimes^{n} \mathbf{H}_{2}=\left[\begin{array}{cc}
\mathbf{H}_{2^{n-1}} & \mathbf{H}_{2^{n-1}} \\
\mathbf{H}_{2^{n-1}} & -\mathbf{H}_{2^{n-1}}
\end{array}\right], n=2,3, \cdots
$$

are called Sylvester Hadamard matrices.

The following lemma is essential to derive our construction methods in the next section. 
Lemma 2: [24] The Kronecker (tensor) product of any two Hadamard matrices is a Hadamard matrix.

\section{Transform Domain Construction Methods}

We first review some transform domain properties of sequences and their correlation functions. A class of ZCZ sequence construction approaches based on transform domain properties is then presented. Detailed comparisons with two related proposals are made and a few construction examples are provided.

\section{A. Useful Transform Domain Properties}

Denote by $\operatorname{DFT}\{u(n)\}$, the DFT of a periodic sequence $\{u(n)\}$ and by $\operatorname{IDFT}\{U(k)\}$, the inverse DFT (IDFT) of a periodic transform domain sequence $\{U(k)\}$. We then immediately have

Lemma 3: The DFT of the CC function $\theta_{u v}(\tau)$ of two period- $N$ sequences, $\{u(n)\}$ and $\{v(n)\}$, is equal to $U(k) V^{*}(k)$, where $\{U(k)\}=\operatorname{DFT}\{u(n)\}$ and $\{V(k)\}=\operatorname{DFT}\{v(n)\}$.

Since the AC function of $\{u(n)\}$ can be expressed as $\theta_{u u}(n)=u(n) \circledast u^{*}(-n)$, its DFT is given by $\Theta_{u u}(k)=|U(k)|^{2}$. Therefore, it is straightforward to show

Corollary 1: Sequence $\{u(n)\}$ is a perfect sequence if and only if $|U(k)|^{2}$ is constant for all $k$.

Based on the above properties, we can easily prove that

Lemma 4: The $\mathrm{AC}$ and $\mathrm{CC}$ functions of a set of sequences are invariant (up to a scaling factor) to filtering if the filtering sequence $v$ is a perfect sequence.

As will become clear in subsequent sections that this lemma makes the filtering operator very useful in transforming a sequence set into one with entries of the sequences taken from a desired constellation while maintaining the correlation properties.

\section{B. Basic Constructions}

Definition 10: A sequence $\{u(n)\}$ in an $(N, K)$ sequence set is said to have a subperiod of $J$, where $J \mid N$, if it is also periodic with period $J<N$, i.e., $u(n)=u(\ell J+n)$, for $0 \leq \ell<N / J$ 
and $0 \leq n<J$

Now note that Lemma 3 implies

$$
\theta_{u v}(\tau)=\sum_{k=0}^{N-1} \Theta_{u v}(k) e^{\frac{j 2 \pi \tau k}{N}}=\sum_{k=0}^{N-1} U(k) V^{*}(k) e^{\frac{j 2 \pi \tau k}{N}}
$$

where $\Theta_{u v}(k)=\operatorname{DFT}\left\{\theta_{u v}(\tau)\right\}$. When $\{U(k)\}$ and $\{V(k)\}$, regarded as $N$-dimensional vectors, are orthogonal, we have

$$
\theta_{u v}(0)=\sum_{k=0}^{N-1} \Theta_{u v}(k)=\sum_{k=0}^{N-1} U(k) V^{*}(k)=0 .
$$

If the sequence $\left\{\Theta_{u v}(k)\right\}$ has a subperiod of $J=\frac{N}{m}$, then

$$
\begin{aligned}
\theta_{u v}(\tau) & =\sum_{k=0}^{J-1} \Theta_{u v}(k) e^{\frac{j 2 \pi \tau k}{N}}+\sum_{k=J}^{2 J-1} \Theta_{u v}(k) e^{\frac{j 2 \pi \tau k}{N}}+\cdots+\sum_{k=(m-1) J}^{N-1} \Theta_{u v}(k) e^{\frac{j 2 \pi \tau k}{N}} \\
& =\sum_{k=0}^{J-1} \Theta_{u v}(k) e^{\frac{j 2 \pi \tau k}{N}}\left(1+e^{\frac{j 2 \pi \tau}{m}}+\cdots+e^{\frac{j 2 \pi \tau(m-1)}{m}}\right)
\end{aligned}
$$

The identity

$$
1+\alpha+\alpha^{2}+\cdots+\alpha^{m-1}=0, \forall \alpha=W_{m}^{\tau},|\tau|_{m} \neq 0
$$

then gives

Lemma 5: The CC function $\theta_{u v}(\tau)$ of two period- $N$ sequences $\{u(n)\}$ and $\{v(n)\}$ is identical zero $\forall|\tau|_{N} \leq T$ if the associated DFT vectors $\{U(k)\}$ and $\{V(k)\}$ are orthogonal and their Hadamard product, $\left\{U(k) V^{*}(k)\right\}$, has a subperiod of $J=N /(T+1)$, where $T$ is a positive integer.

The recursive Kronecker construction of the Sylvester Hadamard matrices (9) gives at least two sets of row vectors (i.e., upper- and lower-half parts of $\mathbf{H}_{2^{n}}$ ) that satisfy both the orthogonality and subperiodicity requirements. This property still holds when we replace Sylvester Hadamard matrices by other classes of Hadamard matrices produced by a recursive Kronecker construction similar to (9). Furthermore, as elements of a Hadamard matrix have constant modulus, the AC of all sequences derived by taking IDFT on rows of a Hadamard matrix is 0 for all nonzero 
correlation lags by Corollary 1. These two observations suggest that ZCZ families can be obtained by using proper subsets of row vectors from a Hadamard matrix. To have a precise definition of "proper subsets," we need

Definition 11: A regular $p$ th-order $M$-partition on an $N \times N$ matrix $\mathbf{H}$, where $N=M^{n}$, is the set of $m=N / K=M^{p} K \times N$ submatrices, each is formed by non-overlapping $K=M^{n-p}$ consecutive rows of $\mathbf{H}$.

Proper subsets of row vectors that generate ZCZ families are obtained by performing $p$ th-order $M$-partition on the $n$th Kronecker power of a Hadamard matrix, i.e.,

Lemma 6: Let $\mathbf{U}$ be a Hadamard matrix of order $M$ and $\mathbf{H}$ be the Hadamard matrix of order $N$ generated by the $n$th Kronecker power of $\mathbf{U}$, i.e.,

$$
\mathbf{H}=\left[\mathbf{h}_{0}^{T}, \mathbf{h}_{1}^{T}, \cdots, \mathbf{h}_{N-1}^{T}\right]^{T}=\otimes^{n} \mathbf{U}
$$

where $N=M^{n}, n \geq 2$, and $\mathbf{h}_{\ell}$ is the $\ell$ th row $^{1}$ of $\mathbf{H}$. We perform a regular $p$ th-order $M$-partition on $\mathbf{H}$ to obtain the $m=M^{p}$ submatrices

$$
\widetilde{\mathbf{H}}_{i}=\left[\mathbf{h}_{i K}^{T}, \cdots, \mathbf{h}_{(i+1) K-1}^{T}\right]^{T}, i=0,1, \cdots, m-1 .
$$

Then, for each $i$, the set of $K$ length- $N$ sequences $\mathbf{A}_{i} \stackrel{\text { def }}{=}\left\{A_{i, 0}, A_{i, 1}, \cdots, A_{i, K-1}\right\}$, where $A_{i, j}=$ $\operatorname{IDFT}\left\{\mathbf{h}_{i K+j}\right\}$, is an $(N, K, m-1) \mathrm{ZCZ}$ sequence family that achieves the upper bound (4). Furthermore, all member sequences in the family are perfect sequences.

Proof: The matrix $\mathbf{H}$ can be expressed in the stacked form, $\mathbf{H}=\left[\widetilde{\mathbf{H}}_{0}^{T}, \widetilde{\mathbf{H}}_{1}^{T}, \cdots, \widetilde{\mathbf{H}}_{m-1}^{T}\right]^{T}$, where the submatrix $\widetilde{\mathbf{H}}_{i}$ is of the form

$$
\left[a_{i, 0} \mathbf{B}, a_{i, 1} \mathbf{B}, \cdots, a_{i, m-1} \mathbf{B}\right]
$$

where $a_{i, j}$ 's have unit magnitudes and $\mathbf{B}=\otimes^{n-p} \mathbf{U}$. It follows immediately that the Hadamard products of two distinct rows of $\widetilde{\mathbf{H}}_{i}$ has a period of $M^{n-p}=K$.

\footnotetext{
${ }^{1}$ For convenience, all the column, row, and vector elements' indices start with 0 instead of 1.
} 
The above construction gives ZCZ sequences of length $M^{n}, n \geq 2$. That the upper bound (4) is achieved is a result of our partition method described by Definition 11. The sequence length constraint can be relaxed by using Kronecker construction of Hadamard matrices of different orders. Using Lemma 2 and an argument similar to that in deriving the above lemma, we obtain

Theorem 1: Let $\mathbf{H}$ be the $N \times N$ Hadamard matrix

$$
\mathbf{H}=\left[\mathbf{h}_{0}^{T}, \mathbf{h}_{1}^{T}, \cdots, \mathbf{h}_{N-1}^{T}\right]^{T} \stackrel{\text { def }}{=} \mathbf{U}_{n-1} \otimes \cdots \otimes \mathbf{U}_{0}
$$

where $\mathbf{U}_{k}, k=0,1, \cdots, n-1$, are $M_{k} \times M_{k}$ (not necessarily distinct) Hadamard matrices and $N=\prod_{k=0}^{n-1} M_{k}, n \geq 2$. Partition $\mathbf{H}$ into $m=\frac{N}{K}$ submatrices of size $K \times N$,

$$
\widetilde{\mathbf{H}}_{i}=\left[\mathbf{h}_{i K}^{T}, \cdots, \mathbf{h}_{(i+1) K-1}^{T}\right]^{T}, i=0,1, \cdots, m-1,
$$

each formed by non-overlapping $K=\prod_{k=0}^{n-p-1} M_{k}$ consecutive rows of $\mathbf{H}$ with $p>0$. Then, for each $i$, the set of $K$ period- $N$ sequences $\mathbf{A}_{i} \stackrel{\text { def }}{=}\left\{A_{i, 0}, A_{i, 1}, \cdots, A_{i, K-1}\right\}$, where $A_{i, j}=$ $\operatorname{IDFT}\left\{\mathbf{h}_{i K+j}\right\}$, is an $(N, K, m-1) \mathrm{ZCZ}$ sequence family that achieves the upper bound (4) ${ }^{2}$.

Note that the recursive generation of Hadamard matrices defined by (9) and (13) are special cases of (15), i.e., the above theorem generalize Theorems 1 and 2 of [25].

\section{Polyphase ZCZ Sequences}

The ZCZ sequences generated by the methods described above are not necessary of constant modulus but can be converted into polyphase sequences without altering the desired $\mathrm{AC}$ and CC properties by a proper filtering process; see Definition 4 and Lemma 4. To find the filtering perfect sequences we need the following two properties.

Lemma 7: [26] Let $U$ be a length- $N$ polyphase perfect sequence with entries drawn from the $N$-PSK constellation. Then both IDFT $\{U\}$ and DFT $\{U\}$ are polyphase perfect sequences.

Lemma 8: [27] Let $L$ be a natural number and $N=L^{2}$. Define the length- $N$ polyphase

\footnotetext{
${ }^{2}$ Technically, the theorem is also valid for $p=0$, as the resulting set has a ZCZ width 0 . We will implicitly ignore this trivial case and assume $p>0$ in the subsequent discussion.
} 
sequence $\{u(k)\}$ by

$$
u\left(k_{1} L+k_{2}\right)=W_{L}^{\beta\left(k_{2}\right) k_{1}+r\left(k_{2}\right)}, 0 \leq k_{1}, k_{2}<L,
$$

where $\left\{\beta\left(k_{2}\right): k_{2}=0,1, \cdots, L-1\right\}$ is a permutation of $\{0,1, \cdots, L-1\}$, and $r\left(k_{2}\right)$ is a rational number depending on $k_{2}$. Then the sequences, $\{u(k)\}$,

$$
\left\{e^{j \theta_{k_{2}}} u\left(k_{1} L+k_{2}\right): 0 \leq \theta_{k_{2}}<2 \pi, 0 \leq k_{1}, k_{2}<L\right\}
$$

and

$$
\left\{W_{L}^{\ell k_{1}} u\left(k_{1} L+k_{2}\right): 0 \leq k_{1}, k_{2}<L\right\} \text {, for any integer } \ell \text {, }
$$

are all polyphase perfect sequences.

Based on the above results, we propose a transform domain construction of polyphase ZCZ sequences as follows.

Corollary 2: Let $\mathbf{u}$ be a length- $N$ perfect sequence of the form (17), $N=\prod_{k=0}^{n-1} M_{k}=L^{2}$ for some $L$, and $\widetilde{\mathbf{H}}_{i}$ be the $i$ th submatrix defined by (15) and (16) using $M_{k}$-DFT or $M_{k}$-IDFT matrices $\mathbf{U}_{k}$ 's. Then $\mathbf{C}_{i}=\left\{\operatorname{IDFT}\left\{\mathbf{h}_{i K+n}\right\} \circ \operatorname{IDFT}\{\mathbf{u}\}: 0 \leq n \leq K-1\right\}$ is an $\left(N, K, \frac{N}{K}-1\right)$ bound-achieving polyphase ZCZ sequence set.

Proof: Since the entries in the $n$th row of $\widetilde{\mathbf{H}}_{i}$ render the general expression

$$
[\mathbf{H}]_{i K+n, k_{1} L+k_{2}} \stackrel{\text { def }}{=} h_{i K+n}\left(k_{1} L+k_{2}\right)=e^{j \theta_{k_{2}}(n)} W_{L}^{\ell(n) k_{1}}
$$

for $0 \leq k_{1}, k_{2}<L$, where $\ell(n) \in \mathbb{Z}$ (integers) and $0 \leq \theta_{k_{2}}(n)<2 \pi$, the products $h_{i K+n}(k) u^{*}(k)$ are of the forms (17)-(19) and are integer powers of $W_{N}$. Lemmas 7 and 8 imply that the sequence

$$
\begin{aligned}
C_{i, n}(k) & =\operatorname{IDFT}\left\{h_{i K+n}(k)\right\} \circ \operatorname{IDFT}\{u(k)\} \\
& =\operatorname{IDFT}\left\{h_{i K+n}(k) u^{*}(k)\right\}
\end{aligned}
$$


has polyphase entries. Invoking Theorem 1 and Lemma 4, we conclude that $\left\{C_{i, n}: 0 \leq n<K\right\}$ is an $\left(N, K, \frac{N}{K}-1\right)$ polyphase ZCZ family.

Remark 1: (Polyphase constraint and sequence length selection) Theorem 1 provides a general transform domain approach using Hadamard matrices to construct bound-achieving sets of arbitrary nonprime length ZCZ sequences. In contrast, Corollary 2 focuses on the generation of polyphase sequences and can be regarded as an extension of a special case of the former. The polyphase requirement is satisfied by invoking an additional filtering operation and the use of special Hadamard matrices; see Example 1 in the ensuing subsection. As a result, the choice of the sequence length is limited to perfect squares $\left(N=L^{2}\right)$.

Remark 2: (Nested structure) Every $K \times N$ submatrix $\widetilde{\mathbf{H}}_{i}$ can be further partitioned into $K / K^{\prime}=\prod_{k=n-p^{\prime}}^{n-p-1} M_{k}$ submatrices of size $K^{\prime} \times N$, where $p<p^{\prime}<n, K=\prod_{k=0}^{n-p-1} M_{k}$, and $K^{\prime}=\prod_{k=0}^{n-p^{\prime}-1} M_{k}$ so that each submatrix can be used to construct an $\left(N, K^{\prime}, \frac{N}{K^{\prime}}-1\right) \mathrm{ZCZ}$ sequence set $\mathbf{C}_{i}^{j}$ with larger ZCZ width and $\bigcup_{j=0}^{\frac{K}{K^{\prime}}-1} \mathbf{C}_{i}^{j}=\mathbf{C}_{i}$. This partition can be done in a nested manner, i.e., each subset can be further decomposed to render even smaller sequence subsets or $\widetilde{\mathbf{H}}_{i}$ can be merged with proper neighboring submatrices to construct a larger set.

Remark 3: (Tradeoff between AC and CC) The identity (12) actually gives a stronger CC property than what is specified by the ZCZ width; it implies that the $\mathrm{CC}$ values are identically zero except at $\tau=s(T+1), s \in \mathbb{Z}$. This is still weaker than the constructions of [15] and [16] which yield perfect (zero) $\mathrm{CC}$ at all lags. Perfect $\mathrm{CC}$ is achieved by requiring that each transform domain sequence has sparse nonzero elements and support (set of the nonzero coordinates) disjoint from the supports of all other transform domain sequences. Nevertheless, their AC functions are not as good as ours as all the sequences constructed by our approach are perfect sequences.

Remark 4: (Tradeoff between sequence length and alphabet size) Tsai's approach [15] is more flexible in the choice of sequence length but requires a very large constellation for elements of the sequences. Our approach, on the other hand, requires the smallest constellation and is more flexible than [16] in selecting the sequence length $N$.

We summarize various parameter constraints for our approach, [15], and [16] in Table I. 
TABLE I

TRANSFORM DOMAIN-BASED POLYPHASE ZCZ SEQUENCE SETS

\begin{tabular}{|c|c|c|c|c|}
\hline & Tsai [15] & Brodzik [16] & Corollary 2 \\
\hline Sequence length $N$ & $n_{1} n_{2}$ & \multicolumn{2}{|c|}{$L^{3}$} & $\prod_{k=0}^{n-1} M_{k}=L^{2}$ \\
\hline Set size $K$ & $n_{2}$ & \multicolumn{2}{|c|}{$L$} & $\prod_{k=0}^{n-p-1} M_{k}$ \\
\hline ZCZ width $T$ & $n_{1}-1$ & $L^{2}-1, L$ prime & $L-1, L$ nonprime & $\frac{N}{K}-1$ \\
\hline $\begin{array}{c}\text { Upper-bound (4) } \\
\text { achieved? }\end{array}$ & Yes & Yes & No & Yes \\
\hline $\begin{array}{c}\text { Perfect sequence } \\
\text { used }\end{array}$ & $\begin{array}{c}\text { Length- } n_{1}, \\
n_{P} \text {-phase }\end{array}$ & No explicit use of perfect sequences & Length- $N$ \\
\hline Alphabet size & lcm $\left(N, n_{P}\right)$ & \multicolumn{2}{|c|}{$N$} & $N$ \\
\hline
\end{tabular}

\section{ZCZ Sequence Sets Generated by Transform Domain Approach}

In this subsection, we present some construction examples using the proposed transform domain method. All ZCZ sequences obtained are perfect sequences. To minimize the number of notations, we use $C_{i}$ and $A_{i}$ to denote sequences generated by the methods of Corollary 2 and Theorem 1, respectively. The same notation may refer to different sequences in different examples when there is no danger of ambiguity.

Example 1: (Use of three DFT matrices of unequal dimensions) Partitioning the Hadamard matrix $\mathbf{H}=\mathbf{F}_{6} \otimes \mathbf{F}_{3} \otimes \mathbf{F}_{2}$ into submatrices $\widetilde{\mathbf{H}}_{0}, \widetilde{\mathbf{H}}_{1}, \cdots, \widetilde{\mathbf{H}}_{17}$ and performing IDFT on the rows of $\widetilde{\mathbf{H}}_{10}$, we obtain two sequences

$$
\begin{aligned}
& A_{0}=\left(000 W_{12}^{21} 00000 W_{12}^{7} 00000 W_{12}^{5} 00000 W_{12}^{15} 00000 W_{12}^{1} 00000 W_{12}^{23} 00\right) \\
& A_{1}=\left(000 W_{12}^{15} 00000 W_{12}^{1} 00000 W_{12}^{23} 00000 W_{12}^{21} 00000 W_{12}^{7} 00000 W_{12}^{5} 00\right)
\end{aligned}
$$

To convert them into ones with constant moduli we filter them by the perfect polyphase sequence [15]

$$
\begin{aligned}
U_{36}= & \left(W_{6}^{0} W_{6}^{0} W_{6}^{0} W_{6}^{0} W_{6}^{0} W_{6}^{0} W_{6}^{0} W_{6}^{5} W_{6}^{4} W_{6}^{3} W_{6}^{2} W_{6}^{1} W_{6}^{0} W_{6}^{4} W_{6}^{2} W_{6}^{0} W_{6}^{4} W_{6}^{2}\right. \\
& \left.W_{6}^{0} W_{6}^{3} W_{6}^{0} W_{6}^{3} W_{6}^{0} W_{6}^{3} W_{6}^{0} W_{6}^{2} W_{6}^{4} W_{6}^{0} W_{6}^{2} W_{6}^{4} W_{6}^{0} W_{6}^{1} W_{6}^{2} W_{6}^{3} W_{6}^{4} W_{6}^{5}\right)
\end{aligned}
$$


which satisfies (17). The resulting $(36,2,17)$ bound-achieving ZCZ sequence set consists of

$$
\begin{aligned}
C_{0}=A_{0} \circ U_{36}= & \left(W_{12}^{11} W_{12}^{8} W_{12}^{9} W_{12}^{0} W_{12}^{3} W_{12}^{2} W_{12}^{5} W_{12}^{4} W_{12}^{7} W_{12}^{0} W_{12}^{5} W_{12}^{6} W_{12}^{11} W_{12}^{12} W_{12}^{5} W_{12}^{0} W_{12}^{7} W_{12}^{10}\right. \\
& \left.W_{12}^{5} W_{12}^{8} W_{12}^{3} W_{12}^{0} W_{12}^{9} W_{12}^{2} W_{12}^{11} W_{12}^{4} W_{12}^{1} W_{12}^{0} W_{12}^{11} W_{12}^{6} W_{12}^{5} W_{12}^{12} W_{12}^{11} W_{12}^{0} W_{12}^{1} W_{12}^{10}\right), \\
C_{1}=A_{1} \circ U_{36}= & \left(W_{12}^{5} W_{12}^{8} W_{12}^{3} W_{12}^{0} W_{12}^{9} W_{12}^{2} W_{12}^{11} W_{12}^{4} W_{12}^{1} W_{12}^{0} W_{12}^{11} W_{12}^{6} W_{12}^{5} W_{12}^{12} W_{12}^{11} W_{12}^{0} W_{12}^{1} W_{12}^{10}\right. \\
& \left.W_{12}^{11} W_{12}^{8} W_{12}^{9} W_{12}^{0} W_{12}^{3} W_{12}^{2} W_{12}^{5} W_{12}^{4} W_{12}^{7} W_{12}^{0} W_{12}^{5} W_{12}^{6} W_{12}^{11} W_{12}^{12} W_{12}^{5} W_{12}^{0} W_{12}^{7} W_{12}^{10}\right) .
\end{aligned}
$$

If instead we take IDFT on the rows of the first submatrix $\widetilde{\mathbf{G}}_{0}$ of $\mathbf{G}=\left[\widetilde{\mathbf{G}}_{0}^{T}, \widetilde{\mathbf{G}}_{1}^{T}, \cdots, \widetilde{\mathbf{G}}_{11}^{T}\right]^{T}=$ $\mathbf{F}_{2} \otimes \mathbf{F}_{6} \otimes \mathbf{F}_{3}$ and filter the resulting sequences $\left\{A_{0}, A_{1}, A_{2}\right\}$ through (20), we obtain the boundachieving $(36,3,11)$ set:

$$
\begin{array}{r}
C_{0}=A_{0} \circ U_{36}=\left(W_{6}^{0} W_{6}^{1} W_{6}^{2} W_{6}^{3} W_{6}^{4} W_{6}^{5} W_{6}^{0} W_{6}^{2} W_{6}^{4} W_{6}^{0} W_{6}^{2} W_{6}^{4} W_{6}^{0} W_{6}^{3} W_{6}^{0} W_{6}^{3} W_{6}^{0} W_{6}^{3}\right. \\
\left.W_{6}^{0} W_{6}^{4} W_{6}^{2} W_{6}^{0} W_{6}^{4} W_{6}^{2} W_{6}^{0} W_{6}^{5} W_{6}^{4} W_{6}^{3} W_{6}^{2} W_{6}^{1} W_{6}^{0} W_{6}^{0} W_{6}^{0} W_{6}^{0} W_{6}^{0} W_{6}^{0}\right), \\
C_{1}=A_{1} \circ U_{36}=\left(W_{6}^{0} W_{6}^{5} W_{6}^{4} W_{6}^{3} W_{6}^{2} W_{6}^{1} W_{6}^{0} W_{6}^{0} W_{6}^{0} W_{6}^{0} W_{6}^{0} W_{6}^{0} W_{6}^{0} W_{6}^{1} W_{6}^{2} W_{6}^{3} W_{6}^{4} W_{6}^{5}\right. \\
\left.W_{6}^{0} W_{6}^{2} W_{6}^{4} W_{6}^{0} W_{6}^{2} W_{6}^{4} W_{6}^{0} W_{6}^{3} W_{6}^{0} W_{6}^{3} W_{6}^{0} W_{6}^{3} W_{6}^{0} W_{6}^{4} W_{6}^{2} W_{6}^{0} W_{6}^{4} W_{6}^{2}\right), \\
C_{2}=A_{2} \circ U_{36}=\left(W_{6}^{0} W_{6}^{3} W_{6}^{0} W_{6}^{3} W_{6}^{0} W_{6}^{3} W_{6}^{0} W_{6}^{4} W_{6}^{2} W_{6}^{0} W_{6}^{4} W_{6}^{2} W_{6}^{0} W_{6}^{5} W_{6}^{4} W_{6}^{3} W_{6}^{2} W_{6}^{1}\right. \\
\left.W_{6}^{0} W_{6}^{0} W_{6}^{0} W_{6}^{0} W_{6}^{0} W_{6}^{0} W_{6}^{0} W_{6}^{1} W_{6}^{2} W_{6}^{3} W_{6}^{4} W_{6}^{5} W_{6}^{0} W_{6}^{2} W_{6}^{4} W_{6}^{0} W_{6}^{2} W_{6}^{4}\right) .
\end{array}
$$

Example 2: (Construction based on Kronecker power of a DFT matrix) Let $\mathbf{H}=\mathbf{F}_{3} \otimes \mathbf{F}_{3} \otimes$ $\mathbf{F}_{3} \otimes \mathbf{F}_{3}$ and denote by $\widetilde{\mathbf{H}}_{0}, \widetilde{\mathbf{H}}_{1}, \cdots, \widetilde{\mathbf{H}}_{26}$ the submatrices obtained by performing regular 3rdorder 3-partition on $\mathbf{H}$. Choosing $\widetilde{\mathbf{H}}_{2}$ and performing IDFT on its rows, we obtain sequences $\left\{A_{0}, A_{1}, A_{2}\right\}$. Filtering them by polyphase perfect sequence

$$
\begin{aligned}
U_{81}= & W_{9}^{0} W_{9}^{0} W_{9}^{0} W_{9}^{0} W_{9}^{0} W_{9}^{0} W_{9}^{0} W_{9}^{0} W_{9}^{0} W_{9}^{0} W_{9}^{8} W_{9}^{7} W_{9}^{6} W_{9}^{5} W_{9}^{4} W_{9}^{3} W_{9}^{2} W_{9}^{1} \\
& W_{9}^{0} W_{9}^{7} W_{9}^{5} W_{9}^{3} W_{9}^{1} W_{9}^{8} W_{9}^{6} W_{9}^{4} W_{9}^{2} W_{9}^{0} W_{9}^{6} W_{9}^{3} W_{9}^{0} W_{9}^{6} W_{9}^{3} W_{9}^{0} W_{9}^{6} W_{9}^{3} \\
& W_{9}^{0} W_{9}^{5} W_{9}^{1} W_{9}^{6} W_{9}^{2} W_{9}^{7} W_{9}^{3} W_{9}^{8} W_{9}^{4} W_{9}^{0} W_{9}^{4} W_{9}^{8} W_{9}^{3} W_{9}^{7} W_{9}^{2} W_{9}^{6} W_{9}^{1} W_{9}^{5} \\
& W_{9}^{0} W_{9}^{3} W_{9}^{6} W_{9}^{0} W_{9}^{3} W_{9}^{6} W_{9}^{0} W_{9}^{3} W_{9}^{6} W_{9}^{0} W_{9}^{2} W_{9}^{4} W_{9}^{6} W_{9}^{8} W_{9}^{1} W_{9}^{3} W_{9}^{5} W_{9}^{7}
\end{aligned}
$$




$$
\left.W_{9}^{0} W_{9}^{1} W_{9}^{2} W_{9}^{3} W_{9}^{4} W_{9}^{5} W_{9}^{6} W_{9}^{7} W_{9}^{8}\right)
$$

we obtain

$$
\begin{aligned}
& C_{0}=A_{0} \circ U_{81}=\left(W_{9}^{0} W_{9}^{1} W_{9}^{2} W_{9}^{6} W_{9}^{7} W_{9}^{8} W_{9}^{3} W_{9}^{4} W_{9}^{5} W_{9}^{0} W_{9}^{2} W_{9}^{4} W_{9}^{0} W_{9}^{2} W_{9}^{4} W_{9}^{0} W_{9}^{2} W_{9}^{4}\right. \\
& W_{9}^{0} W_{9}^{3} W_{9}^{6} W_{9}^{3} W_{9}^{6} W_{9}^{0} W_{9}^{6} W_{9}^{0} W_{9}^{3} W_{9}^{0} W_{9}^{4} W_{9}^{8} W_{9}^{6} W_{9}^{1} W_{9}^{5} W_{9}^{3} W_{9}^{7} W_{9}^{2} \\
& W_{9}^{0} W_{9}^{5} W_{9}^{1} W_{9}^{0} W_{9}^{5} W_{9}^{1} W_{9}^{0} W_{9}^{5} W_{9}^{1} W_{9}^{0} W_{9}^{6} W_{9}^{3} W_{9}^{3} W_{9}^{0} W_{9}^{6} W_{9}^{6} W_{9}^{3} W_{9}^{0} \\
& W_{9}^{0} W_{9}^{7} W_{9}^{5} W_{9}^{6} W_{9}^{4} W_{9}^{2} W_{9}^{3} W_{9}^{1} W_{9}^{8} W_{9}^{0} W_{9}^{8} W_{9}^{7} W_{9}^{0} W_{9}^{8} W_{9}^{7} W_{9}^{0} W_{9}^{8} W_{9}^{7} \\
& \left.W_{9}^{0} W_{9}^{0} W_{9}^{0} W_{9}^{3} W_{9}^{3} W_{9}^{3} W_{9}^{6} W_{9}^{6} W_{9}^{6}\right), \\
& C_{1}=A_{1} \circ U_{81}=\left(W_{9}^{0} W_{9}^{7} W_{9}^{5} W_{9}^{6} W_{9}^{4} W_{9}^{2} W_{9}^{3} W_{9}^{1} W_{9}^{8} W_{9}^{0} W_{9}^{8} W_{9}^{7} W_{9}^{0} W_{9}^{8} W_{9}^{7} W_{9}^{0} W_{9}^{8} W_{9}^{7}\right. \\
& W_{9}^{0} W_{9}^{0} W_{9}^{0} W_{9}^{3} W_{9}^{3} W_{9}^{3} W_{9}^{6} W_{9}^{6} W_{9}^{6} W_{9}^{0} W_{9}^{1} W_{9}^{2} W_{9}^{6} W_{9}^{7} W_{9}^{8} W_{9}^{3} W_{9}^{4} W_{9}^{5} \\
& W_{9}^{0} W_{9}^{2} W_{9}^{4} W_{9}^{0} W_{9}^{2} W_{9}^{4} W_{9}^{0} W_{9}^{2} W_{9}^{4} W_{9}^{0} W_{9}^{3} W_{9}^{6} W_{9}^{3} W_{9}^{6} W_{9}^{0} W_{9}^{6} W_{9}^{0} W_{9}^{3} \\
& W_{9}^{0} W_{9}^{4} W_{9}^{8} W_{9}^{6} W_{9}^{1} W_{9}^{5} W_{9}^{3} W_{9}^{7} W_{9}^{2} W_{9}^{0} W_{9}^{5} W_{9}^{1} W_{9}^{0} W_{9}^{5} W_{9}^{1} W_{9}^{0} W_{9}^{5} W_{9}^{1} \\
& \left.W_{9}^{0} W_{9}^{6} W_{9}^{3} W_{9}^{3} W_{9}^{0} W_{9}^{6} W_{9}^{6} W_{9}^{3} W_{9}^{0}\right), \\
& C_{2}=A_{2} \circ U_{81}=\left(W_{9}^{0} W_{9}^{4} W_{9}^{8} W_{9}^{6} W_{9}^{1} W_{9}^{5} W_{9}^{3} W_{9}^{7} W_{9}^{2} W_{9}^{0} W_{9}^{5} W_{9}^{1} W_{9}^{0} W_{9}^{5} W_{9}^{1} W_{9}^{0} W_{9}^{5} W_{9}^{1}\right. \\
& W_{9}^{0} W_{9}^{6} W_{9}^{3} W_{9}^{3} W_{9}^{0} W_{9}^{6} W_{9}^{6} W_{9}^{3} W_{9}^{0} W_{9}^{0} W_{9}^{7} W_{9}^{5} W_{9}^{6} W_{9}^{4} W_{9}^{2} W_{9}^{3} W_{9}^{1} W_{9}^{8} \\
& W_{9}^{0} W_{9}^{8} W_{9}^{7} W_{9}^{0} W_{9}^{8} W_{9}^{7} W_{9}^{0} W_{9}^{8} W_{9}^{7} W_{9}^{0} W_{9}^{0} W_{9}^{0} W_{9}^{3} W_{9}^{3} W_{9}^{3} W_{9}^{6} W_{9}^{6} W_{9}^{6} \\
& W_{9}^{0} W_{9}^{1} W_{9}^{2} W_{9}^{6} W_{9}^{7} W_{9}^{8} W_{9}^{3} W_{9}^{4} W_{9}^{5} W_{9}^{0} W_{9}^{2} W_{9}^{4} W_{9}^{0} W_{9}^{2} W_{9}^{4} W_{9}^{0} W_{9}^{2} W_{9}^{4} \\
& \left.W_{9}^{0} W_{9}^{3} W_{9}^{6} W_{9}^{3} W_{9}^{6} W_{9}^{0} W_{9}^{6} W_{9}^{0} W_{9}^{3}\right)
\end{aligned}
$$

which form an $(81,3,26) \mathrm{ZCZ}$ sequence set that satisfies (4).

Example 3: (Quadriphase sequences derived from a Sylvester Hadamard matrix) Partition the Sylvester Hadamard matrix $\mathbf{H}_{16}$ into four submatrices, $\widetilde{\mathbf{H}}_{0}, \widetilde{\mathbf{H}}_{1}, \widetilde{\mathbf{H}}_{2}, \widetilde{\mathbf{H}}_{3}$, and select the first submatrix, $\widetilde{\mathbf{H}}_{0}=\left[\mathbf{h}_{0}^{T}, \mathbf{h}_{1}^{T}, \mathbf{h}_{2}^{T}, \mathbf{h}_{3}^{T}\right]^{T}$. Filtering the IDFT of $\mathbf{h}_{i}$ by

$$
U_{16}=\left(W_{4}^{0} W_{4}^{0} W_{4}^{0} W_{4}^{0} W_{4}^{0} W_{4}^{3} W_{4}^{2} W_{4}^{1} W_{4}^{0} W_{4}^{2} W_{4}^{0} W_{4}^{2} W_{4}^{0} W_{4}^{1} W_{4}^{2} W_{4}^{3}\right),
$$


for each $i$, we have

$$
\begin{aligned}
& C_{0}=\left(W_{4}^{0} W_{4}^{1} W_{4}^{2} W_{4}^{3} W_{4}^{0} W_{4}^{2} W_{4}^{0} W_{4}^{2} W_{4}^{0} W_{4}^{3} W_{4}^{2} W_{4}^{1} W_{4}^{0} W_{4}^{0} W_{4}^{0} W_{4}^{0}\right), \\
& C_{1}=\left(W_{4}^{0} W_{4}^{3} W_{4}^{2} W_{4}^{1} W_{4}^{0} W_{4}^{0} W_{4}^{0} W_{4}^{0} W_{4}^{0} W_{4}^{1} W_{4}^{2} W_{4}^{3} W_{4}^{0} W_{4}^{2} W_{4}^{0} W_{4}^{2}\right), \\
& C_{2}=\left(W_{4}^{0} W_{4}^{1} W_{4}^{0} W_{4}^{1} W_{4}^{0} W_{4}^{2} W_{4}^{2} W_{4}^{0} W_{4}^{0} W_{4}^{3} W_{4}^{0} W_{4}^{3} W_{4}^{0} W_{4}^{0} W_{4}^{2} W_{4}^{2}\right), \\
& C_{3}=\left(W_{4}^{0} W_{4}^{3} W_{4}^{0} W_{4}^{3} W_{4}^{0} W_{4}^{0} W_{4}^{2} W_{4}^{2} W_{4}^{0} W_{4}^{1} W_{4}^{0} W_{4}^{1} W_{4}^{0} W_{4}^{2} W_{4}^{2} W_{4}^{0}\right),
\end{aligned}
$$

a quadriphase $(16,4,3) \mathrm{ZCZ}$ sequence family that satisfies (4).

Note that if a 3rd-order 2-partition is used instead, we have a set of only two sequences but with a larger ZCZ width, i.e., we obtain a quadriphase $(16,2,7) \mathrm{ZCZ}$ sequence set consisting of $\left\{A_{0} \circ U_{16}, A_{1} \circ U_{16}\right\}$ or $\left\{A_{2} \circ U_{16}, A_{3} \circ U_{16}\right\}$.

\section{Direct Synthesis Method}

\section{A. Preliminaries}

We now present an alternate approach which is capable of generating ZCZ sequences of arbitrary nonprime periods.

Definition 12: A binary (0- and 1-valued) sequence of period $N$ which satisfies the ZCZ width constraint $T$ on its $\mathrm{AC}$ function is called a basic $(N, T)$ sequence.

A basic sequence can be obtained by the simple rule given in

Lemma 9: A binary sequence $B=\left(b_{0}, b_{1}, \cdots, b_{N-1}\right), b_{i} \in\{0,1\}$, is a basic $(N, T)$ sequence if the minimum run length of 0 's is $T$ (in the circular sense), where a run refers to a string of identical symbols and $T$ is also called the minimum spacing of $B$.

\section{B. Synthesis Process}

Two new operations are needed.

Definition 13: A basic $(N, T)$ sequence $B$ with Hamming weight $w_{H}(B)$ can be expressed as the sum (via component-wise addition) of $M$ length- $N$ binary sequences, $\left\{B_{i}\right\}_{i=0}^{M-1}$, with disjoint nonempty supports so that $\sum_{i=0}^{M-1} w_{H}\left(B_{i}\right)=w_{H}(B)$ and $w_{H}\left(B_{i}\right) \geq 1$. The sequence set $\left\{B_{i}\right\}_{i=0}^{M-1}$ is said to be an orthogonal tone decomposition of $B$. 
It is trivial to see that $\left\{B_{i}\right\}_{i=0}^{M-1}$ is a binary $(N, M, T) \mathrm{ZCZ}$ sequence family and each $B_{i}$ is a basic $\left(N, T_{i}\right)$ sequence with $T_{i} \geq T$.

Definition 14: Let $V=(v(0), v(1), \cdots, v(N-1))$ be a length- $N$ binary sequence with Hamming weight $w_{H}(V)=k$ and $\mathbf{U}=\left[u_{i j}\right]$ be any matrix having $k$ columns and arbitrary number of rows $k^{\prime}$. The $V$-upsampled matrix of $\mathbf{U}$ is the $k^{\prime} \times N$ matrix $\mathbf{P}=\left[p_{i j}\right]$ defined by

$$
p_{i j}= \begin{cases}u_{i m}, & j=s_{V}(m), m=0,1, \cdots, k-1 \\ 0, & \text { otherwise }\end{cases}
$$

where $s_{V}(m)=$ the coordinate of sequence $V$ 's $m$ th nonzero entry. We denote the above rowwise nonuniform upsampling operation on $\mathbf{U}$ by $\mathbf{P}=\mathbf{U} \Delta V$.

Obviously, the nonzero entries in all rows of the matrix $\mathbf{P}=\mathbf{U} \triangle V$ are in the same positions. Hence if $V$ is an $(N, T)$ basic sequence constructed by the procedure described in Lemma 9 , then each row has the same minimum spacing $T$ and all $\mathrm{CC}$ (including $\mathrm{AC}$ ) values are zero at $0<\tau \leq T$. Values of all $\mathrm{CC}$ functions at $\tau=0$ are zero when $\mathbf{U}$ is unitary in which case rows of $\mathbf{P}$ all have $\mathrm{ZCZ}$ width $T$. Invoking Lemma 4 , we have

Lemma 10: Let $B$ be a basic $(N, T)$ sequence with $w_{H}(B)=K, \mathbf{B} \stackrel{\text { def }}{=}\left\{B_{i}\right\}_{i=0}^{M-1}$ be an orthogonal tone decomposition of $B, w_{H}\left(B_{i}\right)=k_{i}$, and $\mathbf{U}_{i}, 0 \leq i<M$ be $k_{i} \times k_{i}$ unitary matrices (not necessarily distinct). Then for each $i$, the rows of nonuniform upsampled matrix $\mathbf{P}_{i}=\mathbf{U}_{i} \triangle B_{i}$ constitute an $\left(N, K_{i}, T_{i}\right)$ ZCZ sequence family, where $T_{i} \geq T$ is the minimum spacing of $B_{i}$. Moreover, the rows of all $\mathbf{P}_{i}$ 's constitute an $(N, K, T)$ ZCZ sequence set.

\section{Polyphase ZCZ Sequences}

The above process does not guarantee a constant modulus constellation for the entries of the generated sequences. We need a special class of basic sequences and a suitable perfect sequence to generates polyphase sequence families.

Theorem 2: Let $A^{\prime}=\left\{a_{n}^{\prime}\right\}$ be a length- $N^{\prime}$ perfect $N_{A^{\prime}}$-PSK sequence, where $2 \leq N_{A^{\prime}} \leq 2 N^{\prime}$ and $A$ be the perfect sequence of length $N=N_{r} N^{\prime}$ derived from $N_{r}$-fold upsampling on $A^{\prime}$. An $\left(N, N_{r}, N^{\prime}-1\right)$ or $\left(N, N_{r}, N^{\prime}-2\right)$ ZCZ $\ell$-PSK sequence family, where $\ell=\operatorname{lcm}\left(N_{A^{\prime}}, N_{r}\right)$, 
can be obtained by filtering the rows of $\mathbf{P}=\mathbf{F}_{N_{r}} \triangle B$ by $A$, where $B=\left(b_{0}, b_{1}, \cdots, b_{N-1}\right)$ is the weight $-N_{r}$ basic sequence defined by

$$
b_{i}= \begin{cases}1, & i=k N^{\prime}, k=0,1, \cdots, N_{r}-1 \\ 0, & \text { otherwise }\end{cases}
$$

if $N_{r}$ and $N^{\prime}$ are relatively prime, or by

$$
b_{i}=\left\{\begin{aligned}
1, & i=k N^{\prime}, k=0,1, \cdots, \frac{L_{0}}{N^{\prime}}-1, \text { or } \\
& i=\ell L_{0}+\left(\frac{N}{L_{0}}-\ell\right)+k N^{\prime}, \text { where } \\
& \ell=1,2, \cdots, \frac{N}{L_{0}}-1, \\
& k=0,1, \cdots, \frac{L_{0}}{N^{\prime}}-1 \\
0, & \text { otherwise, }
\end{aligned}\right.
$$

if $\operatorname{gcd}\left(N_{r}, N^{\prime}\right) \neq 1$, where $L_{0}=\operatorname{lcm}\left(N_{r}, N^{\prime}\right)$.

Proof: See Appendix A.

\section{Properties, Constraints, and Comparisons}

The following three properties about the approach described above are easily verifiable.

Remark 5: (Parameter relations) For a fixed $N$ and $K=N_{r}, \mathrm{ZCZ}$ sequence families generated by (23) achieve the upper bound (4) and those generated from (24) satisfy the relation $K(T+1)=$ $N-N_{r}$.

Remark 6: (Nested-like and inter-set properties) The construction described in Lemma 10 results in a nested-like structure similar to that of Remark 2. Instead of decomposing a Hadamard matrix, we decompose a basic sequence of minimum spacing $S$ into several basic sequences of minimum spacing $S^{\prime} \geq S$ and use the latter basic sequences to construct sequence sets whose union constitutes a larger ZCZ set with a ZCZ width smaller than that of individual subset; see the second part of Example 7.

The construction of Theorem 2 needs a special choice of the Hadamard matrix and basic sequence used because of the polyphase requirement. But as a special case of Lemma 10, it still preserve the nested-like structure. In fact, the basic sequences defined by (23) and (24) can be 
cyclically shifted to generate distinct polyphase ZCZ sequence families with the same $(N, K, T)$. The zero CC zone width between a sequence from the set based on $B$ and one from the set based on a circularly-shifted version of $B$ is determined by the $\mathrm{CC}$ function of the two basic sequences used. If, for instance, $\mathbf{C}_{0}=\left\{C_{0,0}, C_{0,1}, \cdots, C_{0, K-1}\right\}$ and $\mathbf{C}_{1}=\left\{C_{1,0}, C_{1,1}, \cdots, C_{1, K-1}\right\}$ are derived from basic sequence $B(n)$ and $B^{\prime}(n)=B\left(\left|n-n^{\prime}\right|_{N}\right)$, respectively, then $\theta_{C_{0, i} C_{1, \ell}}(\tau)=0$, $\forall i, \ell$ and $|\tau|_{N} \leq T^{\prime}$, where $T^{\prime}<T$ is the zero-CC zone width of $\theta_{B, B^{\prime}}(\tau)$. As a result, the set $\mathrm{C}_{0} \cup \mathrm{C}_{1}$ has the $\mathrm{ZCZ}$ width $T^{\prime}<T$; see Example 4 in the next subsection.

Remark 7: (Binary sequences) To generate binary ZCZ sequences one has to use binary Hadamard matrices, which exist for $N_{r}=2^{\ell}, 12 \times 2^{\ell}$, or $20 \times 2^{\ell}$ [24], to replace the $N_{r}$-DFT matrix, $\mathbf{F}_{N_{r}}$, in constructing $\mathbf{P}$ and reduce the required alphabet size to just $\operatorname{lcm}\left(N_{A^{\prime}}, 2\right)=2$; see Examples 9 and 10.

The parameter selection constraints and related properties for our and some related existing methods are given in Table II. We provide more comparisons in the following remarks.

Remark 8: Theorem 2 does not explicitly mention any restriction on the alphabet size. As these constructions need to use a length- $N^{\prime}$ perfect sequence and $N_{r} \times N_{r}$ Hadamard matrices, which do not always exist for all lengths $\left(N^{\prime}\right)$, matrix dimension $\left(N_{r}\right)$ and all constellation sizes $\left(N_{A^{\prime}}\right)$, the $\mathrm{ZCZ}$ width, sequence length, and family size are thus implicitly constrained by the alphabet size.

Remark 9: Tang et al. [8] classifies the ZCZ sequences construction methods into two major categories, i.e., i) those based on complementary sets and ii) those derived from perfect sequences. Our approach belongs to the latter category and generates sequences with length $N=n_{1} n_{2}$, where $n_{1}$ is the length of a perfect sequences. The constructions proposed in [7]-[13] have similar constraints on the sequence length $N$ and those mentioned in the next three remarks.

Remark 10: In [7], an $\left(N, k,\left(n_{1}-2\right) k^{\ell-1}\right)$ set is constructed by using a length- $n_{1}$ perfect sequence, where $n_{1}=k t, k \leq n_{1}$, but $n_{2}$ must be of the form $k^{\ell}, \ell>1$. The interleaving scheme [8] requires that either i) $\operatorname{gcd}\left(n_{1}, n_{2}\right)=1$ or ii) $n_{1} \mid n_{2}$ or $n_{2} \mid n_{1}$ to generate an $\left(N, n_{2}, n_{1}-1\right)$ or $\left(N, n_{2}, n_{1}-2\right)$ ZCZ family. The length constraints in i) is similar to that for the construction (23) while ii) leads to ZCZ families of the same parameters as those by the construction (24) 
except that the latter is only constrained by $\operatorname{gcd}\left(n_{1}, n_{2}\right) \neq 1$.

Remark 11: A length- $N\left(N=n_{1} n_{2}\right)$ Frank-Chu perfect sequence is used in [13] to generate an $\left(N, n_{2}, n_{1}-1\right)$ family. This method also calls for the use of an $n_{2} \times n_{2}$ DFT or binary Hadamard matrix. However, for the case when $n_{1}$ is a perfect square and a DFT (or binary Hadamard) matrix is used, our approach needs an alphabet of size $\operatorname{lcm}\left(n_{2}, \sqrt{n_{1}}\right)$ or $\operatorname{lcm}\left(2, \sqrt{n_{1}}\right)$ instead of $\operatorname{lcm}\left(n_{2}, n_{1}\right), \operatorname{lcm}\left(n_{2}, 2 n_{1}\right)$ or $\operatorname{lcm}\left(2, n_{1}\right)$ required by [13]. Moreover, as [13] is primarily interested in polyphase (nonbinary) sequences, their approach is not applicable for binary set since it requires $n_{1}=2$. Our constructions, on the other hand, can be applied to generate both binary and nonbinary families.

Remark 12: The construction based on (23) generates sequences that possess the same correlation properties as those of the so-called PS sequences [14]. These sequences are boundachieving; they have nonzero $\mathrm{AC}$ values only on subperiodic correlation lags at $\tau=m(T+1)$, $m \in \mathbb{Z}$, and zero $\mathrm{CC}$ for all lags. While the PS sequences require that $\operatorname{gcd}\left(n_{1}, n_{2}\right)=1$, where $n_{1}$ is a perfect square, to construct an $\left(N, n_{2}, n_{1}-1\right)$ family, our method does not impose any constraint on $n_{1}$. Moreover, when $n_{1}$ is a perfect square, our approach can generate sequences, which, for the convenience of reference, are called PS-like sequences, that require a constellation of size $\operatorname{lcm}\left(n_{2}, \sqrt{n_{1}}\right)=N / \sqrt{n_{1}}$ as opposed to $\operatorname{lcm}\left(n_{1}, n_{2}\right)=N$ required by the PS approach [14]. Similarly, we refer to those families derived from (23) using non-perfect square $n_{1}$ as generalized PS sequences for these sequences cannot be generated by the PS method. Some PS-like and generalized PS sequence sets are given in the following subsection.

\section{E. Examples of Direct Synthesized Sequence Sets}

Example 4: (PS-like sequences) Following the procedure described in Theorem 2 with $N_{r}=$ 2, $N^{\prime}=9, B=(100000000100000000)$ and $\mathbf{U}$ being the Sylvester Hadamard matrix $\mathbf{H}_{2}$, we obtain $\mathbf{P}=\mathbf{U} \triangle B=\left[P_{0}^{T}, P_{1}^{T}\right]^{T}$, where

$$
\begin{aligned}
& P_{0}=(1,0,0,0,0,0,0,0,0, \quad 1,0,0,0,0,0,0,0,0), \\
& P_{1}=(1,0,0,0,0,0,0,0,0,-1,0,0,0,0,0,0,0,0) .
\end{aligned}
$$


TABLE II

Polyphase ZCZ SEQUENCE SETS WITH SEQUENCE LENGTH $N=n_{1} n_{2}$ USING $n_{P}$-PSK PERFECT SEQUENCE

\begin{tabular}{|c|c|c|c|c|c|c|c|}
\hline & Torii [7] & \multicolumn{2}{|c|}{ Tang [8] } & Popovic [13] & Park [14] & Theorem 2 \\
\hline $\begin{array}{c}\text { Perfect } \\
\text { sequence } \\
\text { length }\end{array}$ & $\begin{array}{c}n_{1}=k t, \\
k \leq n_{1}\end{array}$ & \multicolumn{2}{|c|}{$n_{1}$} & $n_{1}=k^{2}$ & $n_{1}$ \\
\hline Set size $K$ & $k$ & \multicolumn{2}{|c|}{$n_{2}$} & $n_{2}$ & $n_{2}$ & $n_{2}$ \\
\hline ZCZ width $T$ & $\left(n_{1}-2\right) k^{\ell-1}$ & $n_{1}-1$ & $n_{1}-2$ & $n_{1}-1$ & $n_{1}-1$ & $n_{1}-1$ & $n_{1}-2$ \\
\hline $\begin{array}{c}\text { Constraints on } \\
n_{2}\end{array}$ & $n_{2}=k^{\ell}, \ell>1$ & $\begin{array}{c}\operatorname{gcd}\left(n_{1}, n_{2}\right) \\
=1\end{array}$ & $\begin{array}{c}n_{1} \mid n_{2} \text { or } \\
n_{2} \mid n_{1}\end{array}$ & None & $\begin{array}{c}\operatorname{gcd}\left(n_{1}, n_{2}\right) \\
=1\end{array}$ & $\begin{array}{c}\operatorname{gcd}\left(n_{1}, n_{2}\right) \\
=1\end{array}$ & $\begin{array}{c}\operatorname{gcd}\left(n_{1}, n_{2}\right) \\
\neq 1\end{array}$ \\
\hline $\begin{array}{c}\text { Upper-bound } \\
(4) \text { achieved? }\end{array}$ & No & Yes & No & Yes & Yes & No \\
\hline Alphabet size & $1 \mathrm{~cm}\left(k, n_{P}\right)$ & $\mathrm{lcm}\left(n_{2}, n_{P}\right)$ & $\begin{array}{c}1 \mathrm{~cm}\left(n_{1}, n_{2}\right) \text { or } \\
\operatorname{lcm}\left(2 n_{1}, n_{2}\right)\end{array}$ & $N$ & $1 \mathrm{~cm}\left(n_{2}, n_{P}\right)$ \\
\hline
\end{tabular}

Filtering them by the upsampled perfect sequence $A=\left(W_{3}^{0} 0 W_{3}^{0} 0 W_{3}^{0} 0 W_{3}^{0} 0 W_{3}^{2} 0 W_{3}^{1} 0 W_{3}^{0} 0 W_{3}^{1} 0 W_{3}^{2} 0\right)$, we have

$$
\begin{aligned}
& C_{0,0}=P_{0} \circ A=\left(W_{6}^{0} W_{6}^{2} W_{6}^{2} W_{6}^{0} W_{6}^{4} W_{6}^{0} W_{6}^{0} W_{6}^{0} W_{6}^{4} W_{6}^{0} W_{6}^{2} W_{6}^{2} W_{6}^{0} W_{6}^{4} W_{6}^{0} W_{6}^{0} W_{6}^{0} W_{6}^{4}\right), \\
& C_{0,1}=P_{1} \circ A=\left(W_{6}^{0} W_{6}^{5} W_{6}^{2} W_{6}^{3} W_{6}^{4} W_{6}^{3} W_{6}^{0} W_{6}^{3} W_{6}^{4} W_{6}^{3} W_{6}^{2} W_{6}^{5} W_{6}^{0} W_{6}^{1} W_{6}^{0} W_{6}^{3} W_{6}^{0} W_{6}^{1}\right) .
\end{aligned}
$$

It can be shown that

$$
\theta_{C_{0,0} C_{0,1}}(\tau)=0, \quad\left|\theta_{C_{0,0} C_{0,0}}(\tau)\right|=\left|\theta_{C_{0,1} C_{0,1}}(\tau)\right|=18 \delta\left(\left.|\tau|\right|_{9}\right)
$$

and $\mathbf{C}_{0}=\left\{C_{0,0}, C_{0,1}\right\}$, is an $(18,2,8)$ bound-achieving ZCZ sequence family.

Using cyclically-shifted basic sequences $B^{\prime}(n)=B\left(|n-3|_{18}\right)$ and $B^{\prime \prime}(n)=B\left(|n-6|_{18}\right)$, we obtain two new $(18,2,8) \mathrm{ZCZ}$ sequence sets $\mathbf{C}_{1}=\left\{C_{1,0}, C_{1,1}\right\}$ and $\mathbf{C}_{2}=\left\{C_{2,0}, C_{2,1}\right\}$ whose members are

$$
\begin{aligned}
& C_{1,0}=\left(W_{6}^{0} W_{6}^{0} W_{6}^{4} W_{6}^{0} W_{6}^{2} W_{6}^{2} W_{6}^{0} W_{6}^{4} W_{6}^{0} W_{6}^{0} W_{6}^{0} W_{6}^{4} W_{6}^{0} W_{6}^{2} W_{6}^{2} W_{6}^{0} W_{6}^{4} W_{6}^{0}\right), \\
& C_{1,1}=\left(W_{6}^{3} W_{6}^{0} W_{6}^{1} W_{6}^{0} W_{6}^{5} W_{6}^{2} W_{6}^{3} W_{6}^{4} W_{6}^{3} W_{6}^{0} W_{6}^{3} W_{6}^{4} W_{6}^{3} W_{6}^{2} W_{6}^{5} W_{6}^{0} W_{6}^{1} W_{6}^{0}\right), \\
& C_{2,0}=\left(W_{6}^{0} W_{6}^{4} W_{6}^{0} W_{6}^{0} W_{6}^{0} W_{6}^{4} W_{6}^{0} W_{6}^{2} W_{6}^{2} W_{6}^{0} W_{6}^{4} W_{6}^{0} W_{6}^{0} W_{6}^{0} W_{6}^{4} W_{6}^{0} W_{6}^{2} W_{6}^{2}\right),
\end{aligned}
$$




$$
C_{2,1}=\left(W_{6}^{0} W_{6}^{1} W_{6}^{0} W_{6}^{3} W_{6}^{0} W_{6}^{1} W_{6}^{0} W_{6}^{5} W_{6}^{2} W_{6}^{3} W_{6}^{4} W_{6}^{3} W_{6}^{0} W_{6}^{3} W_{6}^{4} W_{6}^{3} W_{6}^{2} W_{6}^{5}\right) .
$$

It can be shown that $\theta_{B B^{\prime}}(\tau)=\theta_{B B^{\prime \prime}}(\tau)=\theta_{B^{\prime} B^{\prime \prime}}(\tau)=0, \forall|\tau| \leq T^{\prime}=2$ and thus the inter-set zero-CC zone width is 2 . Moreover, the set $\mathbf{C} \stackrel{\text { def }}{=} \bigcup_{i=0}^{2} \mathbf{C}_{i}$ is a bound-achieving $(18,6,2) \mathrm{ZCZ}$ sequence set.

Example 5: (Length-12 PS-like sequences) The set of three PS-like sequences

$$
\begin{aligned}
& P_{0}=\left(W_{3}^{0} 000 W_{3}^{0} 000 W_{3}^{0} 000\right), \\
& P_{1}=\left(W_{3}^{0} 000 W_{3}^{1} 000 W_{3}^{2} 000\right), \\
& P_{2}=\left(W_{3}^{0} 000 W_{3}^{2} 000 W_{3}^{1} 000\right)
\end{aligned}
$$

is generated by using $N_{r}=3, N^{\prime}=4, B=(100010001000)$, and IDFT matrix $\mathbf{U}=\mathbf{F}_{3}^{H}$. Filtering them by $A=(1,0,0,1,0,0,1,0,0,-1,0,0)$, we obtain the ZCZ sequences

$$
\begin{aligned}
& C_{0}=P_{0} \circ A=\left(W_{6}^{0} W_{6}^{0} W_{6}^{0} W_{6}^{3} W_{6}^{0} W_{6}^{0} W_{6}^{0} W_{6}^{3} W_{6}^{0} W_{6}^{0} W_{6}^{0} W_{6}^{3}\right), \\
& C_{1}=P_{1} \circ A=\left(W_{6}^{0} W_{6}^{2} W_{6}^{4} W_{6}^{3} W_{6}^{2} W_{6}^{4} W_{6}^{0} W_{6}^{5} W_{6}^{4} W_{6}^{0} W_{6}^{2} W_{6}^{1}\right), \\
& C_{2}=P_{2} \circ A=\left(W_{6}^{0} W_{6}^{4} W_{6}^{2} W_{6}^{3} W_{6}^{4} W_{6}^{2} W_{6}^{0} W_{6}^{1} W_{6}^{2} W_{6}^{0} W_{6}^{4} W_{6}^{5}\right) .
\end{aligned}
$$

It is verifiable that $\forall i, j, i \neq j$,

$$
\theta_{C_{i} C_{j}}(\tau)=0, \quad\left|\theta_{C_{i} C_{i}}(\tau)\right|=12 \delta\left(|\tau|_{4}\right),
$$

i.e., $\mathbf{C}=\left\{C_{0}, C_{1}, C_{2}\right\}$ is a $(12,3,3)$ bound-achieving $\mathrm{ZCZ}$ sequence set. This set also possesses the same PS sequence correlation properties [14]. Moreover, both (25) and (26) require only $1 / 3$ and $1 / 2$ of the alphabet size required by the original PS construction under the same sequence period constraint.

Example 6: (Generalized PS sequences) Using the method of Theorem 2 with $N_{r}=5, N^{\prime}=$ 3 , the IDFT matrix $\mathbf{U}=\mathbf{F}_{5}^{H}, B=(100100100100100)$, and $A=\left(W_{3}^{0} 0000 W_{3}^{2} 0000 W_{3}^{0} 0000\right)$, 
we obtain

$$
\begin{aligned}
& C_{0}=\left(W_{15}^{0} W_{15}^{5} W_{15}^{0} W_{15}^{0} W_{15}^{5} W_{15}^{0} W_{15}^{0} W_{15}^{5} W_{15}^{0} W_{15}^{0} W_{15}^{5} W_{15}^{0} W_{15}^{0} W_{15}^{5} W_{15}^{0}\right), \\
& C_{1}=\left(W_{15}^{0} W_{15}^{11} W_{15}^{12} W_{15}^{3} W_{15}^{14} W_{15}^{0} W_{15}^{6} W_{15}^{2} W_{15}^{3} W_{15}^{9} W_{15}^{5} W_{15}^{6} W_{15}^{12} W_{15}^{8} W_{15}^{9}\right), \\
& C_{2}=\left(W_{15}^{0} W_{15}^{2} W_{15}^{9} W_{15}^{6} W_{15}^{8} W_{15}^{0} W_{15}^{12} W_{15}^{14} W_{15}^{6} W_{15}^{3} W_{15}^{5} W_{15}^{12} W_{15}^{9} W_{15}^{11} W_{15}^{3}\right), \\
& C_{3}=\left(W_{15}^{0} W_{15}^{8} W_{15}^{6} W_{15}^{9} W_{15}^{2} W_{15}^{0} W_{15}^{3} W_{15}^{11} W_{15}^{9} W_{15}^{12} W_{15}^{5} W_{15}^{3} W_{15}^{6} W_{15}^{14} W_{15}^{12}\right), \\
& C_{4}=\left(W_{15}^{0} W_{15}^{14} W_{15}^{3} W_{15}^{12} W_{15}^{11} W_{15}^{0} W_{15}^{9} W_{15}^{8} W_{15}^{12} W_{15}^{6} W_{15}^{5} W_{15}^{9} W_{15}^{3} W_{15}^{2} W_{15}^{6}\right)
\end{aligned}
$$

which constitute a set of $(15,5,2)$ bound-achieving generalized PS sequences that has the same correlation properties as the original PS sequences, i.e., $\forall i, j, i \neq j$,

$$
\theta_{C_{i} C_{j}}(\tau)=0, \quad\left|\theta_{C_{i} C_{i}}(\tau)\right|=15 \delta\left(|\tau|_{3}\right)
$$

As mentioned before, the PS method [14] can not produce ZCZ sequences of length $N=15$.

Previous examples are constructed by using coprime $N_{r}$ and $N^{\prime}$, we show a set using the construction (24).

Example 7: (Sets based non-coprime parameters and nested-like sets using orthogonal tone decomposition) By choosing $N_{r}=4, N^{\prime}=6$ and upsampling the Sylvester Hadamard $\mathbf{H}_{4}$ by $B=(100000100000010000010000)$, we obtain a $(24,4,4) \mathrm{ZCZ}$ sequence family by filtering each row of $\mathbf{P}=\mathbf{H}_{4} \triangle B$ through $A=\left(W_{12}^{0} 000 W_{12}^{1} 000 W_{12}^{4} 000 W_{12}^{9} 000 W_{12}^{4} 000 W_{12}^{1} 000\right)$ :

$$
\begin{aligned}
C_{0}=P_{0} \circ A= & \left(W_{12}^{0} W_{12}^{3} W_{12}^{11} W_{12}^{8} W_{12}^{11} W_{12}^{8} W_{12}^{0} W_{12}^{3} W_{12}^{8} W_{12}^{11} W_{12}^{11} W_{12}^{8}\right. \\
& \left.W_{12}^{3} W_{12}^{0} W_{12}^{8} W_{12}^{11} W_{12}^{8} W_{12}^{11} W_{12}^{3} W_{12}^{0} W_{12}^{11} W_{12}^{8} W_{12}^{8} W_{12}^{11}\right), \\
C_{1}=P_{1} \circ A= & \left(W_{12}^{0} W_{12}^{3} W_{12}^{5} W_{12}^{2} W_{12}^{11} W_{12}^{8} W_{12}^{6} W_{12}^{9} W_{12}^{8} W_{12}^{11} W_{12}^{5} W_{12}^{2}\right. \\
& \left.W_{12}^{3} W_{12}^{0} W_{12}^{2} W_{12}^{5} W_{12}^{8} W_{12}^{11} W_{12}^{9} W_{12}^{6} W_{12}^{11} W_{12}^{8} W_{12}^{2} W_{12}^{5}\right), \\
C_{2}=P_{2} \circ A= & \left(W_{12}^{0} W_{12}^{9} W_{12}^{11} W_{12}^{2} W_{12}^{11} W_{12}^{2} W_{12}^{0} W_{12}^{9} W_{12}^{8} W_{12}^{5} W_{12}^{11} W_{12}^{2}\right. \\
& \left.W_{12}^{3} W_{12}^{6} W_{12}^{8} W_{12}^{5} W_{12}^{8} W_{12}^{5} W_{12}^{3} W_{12}^{6} W_{12}^{11} W_{12}^{2} W_{12}^{8} W_{12}^{5}\right), \\
C_{3}=P_{3} \circ A= & \left(W_{12}^{0} W_{12}^{9} W_{12}^{5} W_{12}^{8} W_{12}^{11} W_{12}^{2} W_{12}^{6} W_{12}^{3} W_{12}^{8} W_{12}^{5} W_{12}^{5} W_{12}^{8}\right.
\end{aligned}
$$




$$
\left.W_{12}^{3} W_{12}^{6} W_{12}^{2} W_{12}^{11} W_{12}^{8} W_{12}^{5} W_{12}^{9} W_{12}^{0} W_{12}^{11} W_{12}^{2} W_{12}^{2} W_{12}^{11}\right) .
$$

Alternatively, we can perform orthogonal tone decomposition on $B$ to obtain two weight- 2 basic sequences of same minimum spacing 10 :

$$
\begin{aligned}
& B_{0}=(100000000000010000000000), \\
& B_{1}=(000000100000000000010000) .
\end{aligned}
$$

With $\mathbf{U}_{0}=\mathbf{H}_{2}$ and

$$
\mathbf{U}_{1}=\left[\begin{array}{ll}
1 & j \\
j & 1
\end{array}\right],
$$

we filter rows of $\mathbf{U}_{0} \Delta B_{0}$ and $\mathbf{U}_{1} \Delta B_{1}$ by [27]

$$
A=\left(W_{6}^{0} 0 W_{6}^{0} 0 W_{6}^{3} 0 W_{6}^{2} 0 W_{6}^{4} 0 W_{6}^{2} 0 W_{6}^{3} 0 W_{6}^{6} 0 W_{6}^{6} 0 W_{6}^{2} 0 W_{6}^{1} 0 W_{6}^{2} 0\right)
$$

to obtain two smaller polyphase sets of larger ZCZ width, $\mathbf{C}_{0}=\left\{C_{0,0}, C_{0,1}\right\}$ and $\mathbf{C}_{1}=$ $\left\{C_{1,0}, C_{1,1}\right\}$, where

$$
\begin{aligned}
C_{0,0}= & \left(W_{6}^{0} W_{6}^{3} W_{6}^{4} W_{6}^{4} W_{6}^{5} W_{6}^{2} W_{6}^{4} W_{6}^{4} W_{6}^{0} W_{6}^{3} W_{6}^{0} W_{6}^{0}\right. \\
& \left.W_{6}^{3} W_{6}^{0} W_{6}^{4} W_{6}^{4} W_{6}^{2} W_{6}^{5} W_{6}^{4} W_{6}^{4} W_{6}^{3} W_{6}^{0} W_{6}^{0} W_{6}^{0}\right), \\
C_{0,1}= & \left(W_{6}^{0} W_{6}^{0} W_{6}^{4} W_{6}^{1} W_{6}^{5} W_{6}^{5} W_{6}^{4} W_{6}^{1} W_{6}^{0} W_{6}^{0} W_{6}^{0} W_{6}^{3}\right. \\
& \left.W_{6}^{3} W_{6}^{3} W_{6}^{4} W_{6}^{1} W_{6}^{2} W_{6}^{2} W_{6}^{4} W_{6}^{1} W_{6}^{3} W_{6}^{3} W_{6}^{0} W_{6}^{3}\right), \\
C_{1,0}= & \left(W_{12}^{8} W_{12}^{11} W_{12}^{6} W_{12}^{3} W_{12}^{0} W_{12}^{3} W_{12}^{0} W_{12}^{9} W_{12}^{8} W_{12}^{11} W_{12}^{10} W_{12}^{7}\right. \\
& \left.W_{12}^{8} W_{12}^{11} W_{12}^{0} W_{12}^{9} W_{12}^{0} W_{12}^{3} W_{12}^{6} W_{12}^{3} W_{12}^{8} W_{12}^{11} W_{12}^{4} W_{12}^{1}\right), \\
C_{1,1}= & \left(W_{12}^{11} W_{12}^{8} W_{12}^{9} W_{12}^{0} W_{12}^{3} W_{12}^{0} W_{12}^{3} W_{12}^{6} W_{12}^{11} W_{12}^{8} W_{12}^{1} W_{12}^{4}\right. \\
& \left.W_{12}^{11} W_{12}^{8} W_{12}^{3} W_{12}^{6} W_{12}^{3} W_{12}^{0} W_{12}^{9} W_{12}^{0} W_{12}^{11} W_{12}^{8} W_{12}^{7} W_{12}^{10}\right) .
\end{aligned}
$$

Both sets are $(24,2,10)$ ZCZ sequence sets and together they form another $(24,4,4)$ set. 
Example 8: (Generalized PS sequence set) We can also derive a smaller generalized PS sequence set having the same period but a larger ZCZ width. For example, if we choose $N^{\prime}=8$, $N_{r}=3$, (23), and use the QPSK perfect sequence [28]

$$
A^{\prime}=\left(W_{4}^{1} W_{4}^{1} W_{4}^{2} W_{4}^{1} W_{4}^{1} W_{4}^{3} W_{4}^{2} W_{4}^{3}\right)
$$

then the three sequences

$$
\begin{aligned}
C_{0}= & \left(W_{12}^{9} W_{12}^{3} W_{12}^{6} W_{12}^{3} W_{12}^{9} W_{12}^{9} W_{12}^{6} W_{12}^{9} W_{12}^{9} W_{12}^{3} W_{12}^{6} W_{12}^{3}\right. \\
& \left.W_{12}^{9} W_{12}^{9} W_{12}^{6} W_{12}^{9} W_{12}^{9} W_{12}^{3} W_{12}^{6} W_{12}^{3} W_{12}^{9} W_{12}^{9} W_{12}^{6} W_{12}^{9}\right), \\
C_{1}= & \left(W_{12}^{9} W_{12}^{7} W_{12}^{2} W_{12}^{3} W_{12}^{1} W_{12}^{5} W_{12}^{6} W_{12}^{1} W_{12}^{5} W_{12}^{3} W_{12}^{10} W_{12}^{11}\right. \\
& \left.W_{12}^{9} W_{12}^{1} W_{12}^{2} W_{12}^{9} W_{12}^{1} W_{12}^{11} W_{12}^{6} W_{12}^{7} W_{12}^{5} W_{12}^{9} W_{12}^{10} W_{12}^{5}\right), \\
C_{2}= & \left(W_{12}^{9} W_{12}^{11} W_{12}^{10} W_{12}^{3} W_{12}^{5} W_{12}^{1} W_{12}^{6} W_{12}^{5} W_{12}^{1} W_{12}^{3} W_{12}^{2} W_{12}^{7}\right. \\
& \left.W_{12}^{9} W_{12}^{5} W_{12}^{10} W_{12}^{9} W_{12}^{5} W_{12}^{7} W_{12}^{6} W_{12}^{11} W_{12}^{1} W_{12}^{9} W_{12}^{2} W_{12}^{1}\right)
\end{aligned}
$$

constitute an $\left(N, N_{r}, N^{\prime}-1\right)=(24,3,7)$ bound-achieving ZCZ family. A family with such ZCZ parameter values can not be generated by the method suggested in [14].

Example 9: (Length-16 ternary and binary sequences) Using the basic sequence $B=(10000$ 00100100100), the Sylvester Hadamard matrix $\mathbf{H}_{4}$ as $\mathbf{U}$, and the perfect sequence $A=(+000+$ $000+000-000)$, we obtain

$$
\begin{aligned}
& P_{0}=(+000000+00+00+00), \\
& P_{1}=(+000000-00+00-00), \\
& P_{2}=(+000000+00-00-00), \\
& P_{3}=(+000000-00-00+00),
\end{aligned}
$$

where + and - denote +1 and -1 , respectively. Time domain sequences with zero entries are often undesirable as they require on-off switching. Filtering $\left\{P_{i}\right\}$ by $A$, we obtain the binary 
$(16,4,2) \mathrm{ZCZ}$ sequence family consisting of

$$
\begin{aligned}
& C_{0}=P_{0} \circ A=(+-++-++++++-++-+), \\
& C_{1}=P_{1} \circ A=(+++---+-+-+++---), \\
& C_{2}=P_{2} \circ A=(++-+---++---+-++), \\
& C_{3}=P_{3} \circ A=(+----+--++-++++-) .
\end{aligned}
$$

Example 10: (Length-32 binary sequence set) Let $N_{r}=8$ and $N^{\prime}=4$. With $B=(1000$ $1000000100010010001001000100), A=(+0000000+0000000+0000000-0000000)$ and $\mathbf{U}=\mathbf{H}_{8}$, we obtain the binary $(32,8,2)$ ZCZ sequence set

$$
\begin{aligned}
& C_{0}=(+-+++-++-+++-++++++-+++-++-+++-+), \\
& C_{1}=(+-++-+---++++---+++----+++-+--+-), \\
& C_{2}=(+++-+++---+---+-+-+++-+++---+---), \\
& C_{3}=(+++----+--+-++-++-++-+--+----+++), \\
& C_{4}=(++-+++-+---+---++---+---+-+++-++), \\
& C_{5}=(++-+--+----++++-+----++++-++-+--), \\
& C_{6}=(+---+----+---+--++-+++-++++-+++-), \\
& C_{7}=(+----+++-+--+-++++-+--+-+++----+) .
\end{aligned}
$$

The ZCZ families shown in the above two examples achieve (5), the bound for binary $\left(N_{A^{\prime}}=\right.$ 2) sequences, but their ZCZ widths are limited by the facts that there exists only one binary perfect sequence (whose length $N^{\prime}=4$ ) and binary Hadamard matrices only exists for certain $N_{r}$; see Remark 7. To increase the ZCZ width and have greater flexibility in choosing the ZCZ parameters, we can use higher-order constellations $\left(N_{r}>2\right)$. For example, quadriphase perfect 
sequences of length $N^{\prime}=2,4,8$ or 16 do exist [27], [28]. We introduce in the next section an alternate method which offers more choices for the ZCZ width.

\section{Sequences Derived from Complementary Sets of Sequences}

In this section, we generalize the above basic sequence based approach by replacing rows of an unitary matrix with concatenated sequences. The following definitions can be found in [22].

\section{A. Basic Definitions}

Definition 15: The aperiodic CC function of two length- $L$ sequences $u \equiv\{u(n)\}$ and $v \equiv$ $\{v(n)\}$ is defined as

$$
\psi_{u v}(\tau)=\sum_{n=\tau}^{L-1} u(n) v^{*}(n-\tau)
$$

The aperiodic AC function of sequence $u$ is obviously $\psi_{u u}(\tau)$.

Definition 16: A set of $Q$ equal-length sequences, $\mathbf{E}=\left\{E_{0}, E_{1}, \cdots, E_{Q-1}\right\}$, forms a complementary set of sequences (CSS) if and only if $\forall \tau \neq 0$,

$$
\sum_{i=0}^{Q-1} \psi_{E_{i} E_{i}}(\tau)=0 .
$$

Definition 17: A CSS, $\mathbf{F}=\left\{F_{0}, F_{1}, \cdots, F_{Q-1}\right\}$, is said to be a mate of the CSS, $\mathbf{E}=$ $\left\{E_{0}, E_{1}, \cdots, E_{Q-1}\right\}$ if

(a) The lengths of all members in $\mathbf{E}$ and $\mathbf{F}$ are the same;

(b) For all $\tau$,

$$
\sum_{i=0}^{Q-1} \psi_{E_{i} F_{i}}(\tau)=0
$$

Definition 18: A collection of complementary sets of sequences $\left\{\mathbf{E}_{0}, \mathbf{E}_{1}, \cdots, \mathbf{E}_{K-1}\right\}$, where each set contains the same number of sequences, is said to be mutually orthogonal if every two sets in the collection are mates of each other.

It has been proved in [21] that 
Corollary 3: The number of mutually orthogonal CSS's (MOCSS's) $K$ cannot exceed the cardinality of member CSS, $Q$, i.e., $K \leq Q$.

\section{B. Synthesis Procedure}

We now extend the nonuniform upsampling operation defined in Definition 14.

Definition 19: Let $V$ be a length- $N$ binary sequence with $w_{H}(V)=Q$ and $\mathcal{E}=\left\{\mathbf{E}_{0}, \mathbf{E}_{1}, \cdots, \mathbf{E}_{K-1}\right\}$ be a collection of $K$ MOCSS's in which each CSS $\mathbf{E}_{i}$ consists of $Q$ length- $L$ sequences, i.e., $\mathbf{E}_{i}=\left\{E_{i, 0}, E_{i, 1}, \cdots, E_{i, Q-1}\right\}$, where $E_{i, j}=\left(e_{i, j}(0), e_{i, j}(1), \cdots, e_{i, j}(L-1)\right)$.

The $V$-upsampled concatenated sequence based on $\mathbf{E}_{i}, G_{i}=\mathbf{E}_{i} \triangle_{c} V=\left(g_{i}(0), g_{i}(1), \cdots, g_{i}(N+\right.$ $Q(L-1)-1))$ is defined by

$$
g_{i}(n)= \begin{cases}e_{i, j}(m), & n=j(L-1)+s_{V}(j)+m \\ 0, & \text { otherwise }\end{cases}
$$

where $s_{V}(j)$ is given in Definition 14 .

The operator $\Delta_{c}$ is similar to $\triangle$ : the latter operates on rows of a matrix while the former operates on the sequence formed by concatenating members of the set $\mathbf{E}_{i}$ and replaces each nonzero element of a basic sequence by a finite-length sequence.

Lemma 11: Let $\mathcal{E}=\left\{\mathbf{E}_{0}, \mathbf{E}_{1}, \cdots, \mathbf{E}_{K-1}\right\}$ be a collection of $K$ MOCSS's in which each set $\mathbf{E}_{i}$ has $Q$ length- $L$ sequences and $B$ be a basic $(N, T)$ sequence of weight $Q$. The set $\mathbf{G}=\left\{\mathbf{E}_{i} \triangle_{c} B\right\} \stackrel{\text { def }}{=}\left\{G_{0}, G_{1}, \cdots, G_{K-1}\right\}$ forms an $(N+Q(L-1), K, T)$ ZCZ sequence family.

Proof: Based on Lemma 9 and Definition 19 we can express $G_{i}$ as

$$
\begin{gathered}
G_{i}=(\underbrace{0 \cdots 0}_{s_{V}(0)} \underbrace{e_{i, 0}(0) \cdots e_{i, 0}(L-1)}_{L} \underbrace{0 \cdots 0}_{s_{V}(1)-s_{V}(0)-1} \\
\underbrace{e_{i, 1}(0) \cdots e_{i, 1}(L-1)}_{s_{V}(2)-s_{V}(1)-1} \underbrace{0 \cdots 0}_{L} \\
\underbrace{e_{i, Q-1}(0) \cdots e_{i, Q-1}(L-1)}_{L} \overbrace{0 \cdots 0}^{N-s_{V}(Q-1)-1}),
\end{gathered}
$$


where $s_{V}(j)-s_{V}(j-1)-1 \geq T, j=0,1, \cdots, Q-1$, and $s_{V}(0)-s_{V}(Q-1)+N-1 \geq T$. Invoking Definitions 17 and 18 , we obtain, for all $i \neq k$,

$$
\theta_{G_{i} G_{k}}(\tau)=\sum_{j=0}^{Q-1} \psi_{E_{i, j} E_{k, j}}(\tau)=0,|\tau|_{N} \leq T .
$$

By analogy, Definition 16 gives, for all $i, \theta_{G_{i} G_{i}}(\tau)=\sum_{j=0}^{Q-1} \psi_{E_{i, j} E_{i, j}}(\tau)=0,0<|\tau|_{N} \leq T$. Therefore, $\mathbf{G}$ forms an $(N+Q(L-1), K, T) \mathrm{ZCZ}$ sequence set.

\section{Polyphase ZCZ Sequences}

Following the idea described in Section IV-C, we can derive another class of polyphase ZCZ sequence families by using suitable perfect and basic sequences. The proof of the next corollary is similar to that of Theorem 2 and is given in the last two paragraphs of Appendix A.

Corollary 4: Let $A$ be the length- $L N$ perfect sequence obtained by $L N_{r}$-fold upsampling on a length- $N^{\prime}$ perfect $N_{A^{\prime}}$-PSK sequence, $A^{\prime}$, where $N=N_{r} N^{\prime}$ and $2 \leq N_{A^{\prime}} \leq N^{\prime}$. Denote by $\mathcal{E}=\left\{\mathbf{E}_{0}, \mathbf{E}_{1}, \cdots, \mathbf{E}_{K-1}\right\}$ a collection of $K$ MOCSS's, where $K \leq N_{r}$ and each CSS $\mathbf{E}_{i}=$ $\left\{E_{i, 0}, E_{i, 1}, \cdots, E_{i, N_{r}-1}\right\}$ contains $N_{r}$ length- $L N_{c}$-PSK sequences. An $(L N, K, T)$ ZCZ $M$-PSK sequence set, $M=\operatorname{lcm}\left(N_{A^{\prime}}, N_{c}\right)$, with $T=L\left(N^{\prime}-2\right)$ if $\operatorname{gcd}\left(N_{r}, N^{\prime}\right) \neq 1$ or $T=L\left(N^{\prime}-1\right)$ if $\operatorname{gcd}\left(N_{r}, N^{\prime}\right)=1$ can be obtained by the following steps:

1) Generate $K$ length- $\left(N+N_{r}(L-1)\right)$ sequences $G_{i}^{\prime}=\mathbf{E}_{i} \triangle_{c} B, i=0,1, \cdots, K-1$, where $B$ is the weight $-N_{r}$ basic sequence of length $N$ defined by (24) if $\operatorname{gcd}\left(N_{r}, N^{\prime}\right) \neq 1$ or by (23) if $\operatorname{gcd}\left(N_{r}, N^{\prime}\right)=1$.

2) Replace each zero in $G_{i}^{\prime}$ by a length- $L$ all-zero sequence to obtain the augmented sequence $G_{i}$

3) Filter each $G_{i}$ by $A$.

We have the following four remarks on the MOCSS-based approach.

Remark 13: Similar to Remark 6, the basic sequence $B$ can be cyclically shifted to generate different polyphase ZCZ families with the same ZCZ parameters and alphabet size. These families can be combined to form a larger family with smaller ZCZ width. Likewise, the zero-CC zone 
width between $B$ and its shifted version $B^{\prime}$ determine the inter-set zero-CC zone width between the associated families or the ZCZ width of the combined set. We can also decompose $B$ into several basic sequences $\left\{B_{i}\right\}$ to generate multiple sets with different ZCZ widths.

Remark 14: As mentioned in Section IV, binary sequence sets constructed by Theorem 2 have less choices in ZCZ width. The construction described in Corollary 4 takes advantage of the fact that the member sequence of an MOCSS exists for many values of $L$ and thus allow the ZCZ width to be chosen from the set $\{T=2 L\}$ with the same basic sequence $B$, set cardinality $N_{r}$, and perfect sequence $A^{\prime}$.

Remark 15: [4] and [5] present MOCSS-based methods for generating binary ZCZ sequences. The approach given in [5] was later generalized by [6]. The ZCZ parameters realizable by these methods can be obtained by using our approach described above. For example, a method given in [4] needs to use a class of recursively generated families of binary $\operatorname{CSS}\left\{\boldsymbol{\Delta}_{n}\right\}$. Expressing a family of $Q$ MOCSS's in matrix form [22]

$$
\boldsymbol{\Delta}_{1} \stackrel{\text { def }}{=}\left[\begin{array}{cccc}
E_{0,0} & E_{1,0} & \cdots & E_{Q-1,0} \\
E_{0,1} & E_{1,1} & \cdots & E_{Q-1,1} \\
\vdots & \vdots & \ddots & \vdots \\
E_{0, Q-1} & E_{1, Q-1} & \cdots & E_{Q-1, Q-1}
\end{array}\right]
$$

where $E_{i, j}$ are length- $L$ binary sequences and each row is a CSS. Then, for $n \geq 2$,

$$
\boldsymbol{\Delta}_{n}=\left[\begin{array}{cc}
\boldsymbol{\Delta}_{n-1} \diamond \boldsymbol{\Delta}_{n-1} & -\boldsymbol{\Delta}_{n-1} \diamond \boldsymbol{\Delta}_{n-1} \\
-\boldsymbol{\Delta}_{n-1} \diamond \boldsymbol{\Delta}_{n-1} & \boldsymbol{\Delta}_{n-1} \diamond \boldsymbol{\Delta}_{n-1}
\end{array}\right],
$$

where $[\mathbf{A} \diamond \mathbf{B}]_{i j}$, the $(i, j)$ th entry of the submatrix $[\mathbf{A} \diamond \mathbf{B}]$, is obtained by concatenating the two sequences, $[\mathbf{A}]_{i j}$ and $[\mathbf{B}]_{i j}$. The concatenation of rows of $\boldsymbol{\Delta}_{n}$ forms a $\left(4^{n-1} L Q, 2^{n-1} Q, 2^{n-2} L\right) \mathrm{ZCZ}$ sequence set. On the other hand, by using $A^{\prime}=(1,1,1,-1)$, the basic sequence defined by (24) and the family of MOCSS $\boldsymbol{\Delta}_{n}$ with $N_{r}=2^{n-1} Q$ and elements of $\boldsymbol{\Delta}_{1}$ being length $L / 4$ sequences, we obtain binary ZCZ sequence sets with the parameters $\left(4^{n-1} 4(L / 4) Q, 2^{n-1} Q, 2^{n-1}(L / 4) \cdot 2\right)=$ $\left(4^{n-1} L Q, 2^{n-1} Q, 2^{n-2} L\right)$ via Corollary 4 . 
TABLE III

ZCZ SEQUENCE SETS (EACH USES A COLLECTION OF $M$ MOCSS's OF $Q$ LENGTH- $L$ SEQUENCES

\begin{tabular}{|c|c|c|c|c|}
\hline & \multicolumn{3}{|c|}{ Deng [4] and Tang [6], $n \geq 0$} & Corollary 4 \\
\hline Sequence length $N$ & $4^{n} L Q$ & $2^{2 n-1} L Q$ & $2 L Q$ & $4 L Q$ \\
\hline Set size $K$ & $2^{n} M$ & $2^{n} M$ & $M$ & $M$ \\
\hline ZCZ width $T$ & $2^{n-1} L$ & $2^{n-2} L$ & $L$ & $2 L$ \\
\hline $\begin{array}{c}\text { Upper-bound (5) } \\
\text { achieved with } \\
M=Q \text { ? }\end{array}$ & Yes & Yes & Yes & Yes \\
\hline Alphabet Size & Binary & $\begin{array}{c}\text { Binary and } \\
\text { polyphase }\end{array}$ \\
\hline
\end{tabular}

Remark 16: Our approach offers more choices in parameter values and thus produce sets which are not derivable from the methods of [4], [6]. More importantly, we can generate not only binary but also nonbinary sequences and the ZCZ parameters for the nonbinary class can be flexibly controlled via $N^{\prime}$, which can be any integer and is not affected by the MOCSS chosen. In Table III we list key parameters for our and some other MOCSS-based binary ZCZ sequence set constructions.

\section{Examples of CSS-Based Polyphase ZCZ Sequence Sets}

Two ZCZ sequence construction examples based on CSS are given in this subsection.

Example 11: $\left(\operatorname{gcd}\left(N_{r}, N^{\prime}\right) \neq 1\right)$ Let $N=16, N^{\prime}=N_{r}=K=L=4, A^{\prime}=(+++-)$, and $B=(1000000100100100)$ and choose a collection of mutually orthogonal complementary sets $\mathcal{E}=\left\{\mathbf{E}_{0}, \mathbf{E}_{1}, \mathbf{E}_{2}, \mathbf{E}_{3}\right\}$ from [22], where

$$
\begin{aligned}
& \mathbf{E}_{0}=\{(++++),(--++),(-+-+),(+--+)\}, \\
& \mathbf{E}_{1}=\{(++--),(----),(-++-),(+-+-)\}, \\
& \mathbf{E}_{2}=\{(-+-+),(+--+),(++++),(--++)\}, \\
& \mathbf{E}_{3}=\{(-++-),(+-+-),(++--),(----)\} .
\end{aligned}
$$

Following the procedure of Corollary 4, we obtain the bound-achieving binary $(64,4,8) \mathrm{ZCZ}$ 
sequence set:

$$
\begin{aligned}
C_{0}= & (+++-++--+-+-++---+--++-+--++ \\
& +++++--+-+-+++--+++++--++-+---++), \\
C_{1}= & (++---+-+-++------+++-+--++---- \\
& \quad++-+-+--++-++++++--+-+-+--+----), \\
& \quad(-+-+++--+++++--++-+---+++++++--+ \\
C_{2}= & \quad+-+--++++++-++--+-+--++----+--+), \\
& \quad(-++-++++++--+-+-+--+----++--+-+- \\
C_{3}= &
\end{aligned}
$$

With the same $B, A^{\prime}$, and $N_{r}$ as those used in Example 10, this set extends the ZCZ width without changing the set cardinality.

Example 12: $\left(\operatorname{gcd}\left(N_{r}, N^{\prime}\right)=1\right)$ Using the construction (23) with (36), $A^{\prime}=\left(W_{3}^{0} W_{3}^{2} W_{3}^{0}\right)$, $N^{\prime}=3, N_{r}=4$, and $L=4$, we can obtain a ZCZ sequence set $\mathrm{C}$ of the same (or larger) $T$ with a shorter sequence period $L N$ and slightly larger constellation:

$$
\begin{aligned}
C_{0}= & \left(W_{6}^{0} W_{6}^{0} W_{6}^{0} W_{6}^{0} W_{6}^{0} W_{6}^{3} W_{6}^{3} W_{6}^{0} W_{6}^{5} W_{6}^{2} W_{6}^{5} W_{6}^{2} W_{6}^{3} W_{6}^{3} W_{6}^{0} W_{6}^{0} W_{6}^{0} W_{6}^{0} W_{6}^{0} W_{6}^{0} W_{6}^{2} W_{6}^{5} W_{6}^{5} W_{6}^{2}\right. \\
& \left.W_{6}^{3} W_{6}^{0} W_{6}^{3} W_{6}^{0} W_{6}^{3} W_{6}^{3} W_{6}^{0} W_{6}^{0} W_{6}^{2} W_{6}^{2} W_{6}^{2} W_{6}^{2} W_{6}^{0} W_{6}^{3} W_{6}^{3} W_{6}^{0} W_{6}^{3} W_{6}^{0} W_{6}^{3} W_{6}^{0} W_{6}^{5} W_{6}^{5} W_{6}^{2} W_{6}^{2}\right), \\
C_{1}= & \left(W_{6}^{0} W_{6}^{0} W_{6}^{3} W_{6}^{3} W_{6}^{0} W_{6}^{3} W_{6}^{0} W_{6}^{3} W_{6}^{5} W_{6}^{2} W_{6}^{2} W_{6}^{5} W_{6}^{3} W_{6}^{3} W_{6}^{3} W_{6}^{3} W_{6}^{0} W_{6}^{0} W_{6}^{3} W_{6}^{3} W_{6}^{2} W_{6}^{5} W_{6}^{2} W_{6}^{5}\right. \\
& \left.W_{6}^{3} W_{6}^{0} W_{6}^{0} W_{6}^{3} W_{6}^{3} W_{6}^{3} W_{6}^{3} W_{6}^{3} W_{6}^{2} W_{6}^{2} W_{6}^{5} W_{6}^{5} W_{6}^{0} W_{6}^{3} W_{6}^{0} W_{6}^{3} W_{6}^{3} W_{6}^{0} W_{6}^{0} W_{6}^{3} W_{6}^{5} W_{6}^{5} W_{6}^{5} W_{6}^{5}\right), \\
C_{2}= & \left(W_{6}^{3} W_{6}^{0} W_{6}^{3} W_{6}^{0} W_{6}^{3} W_{6}^{3} W_{6}^{0} W_{6}^{0} W_{6}^{2} W_{6}^{2} W_{6}^{2} W_{6}^{2} W_{6}^{0} W_{6}^{3} W_{6}^{3} W_{6}^{0} W_{6}^{3} W_{6}^{0} W_{6}^{3} W_{6}^{0} W_{6}^{5} W_{6}^{5} W_{6}^{2} W_{6}^{2}\right. \\
& \left.W_{6}^{0} W_{6}^{0} W_{6}^{0} W_{6}^{0} W_{6}^{0} W_{6}^{3} W_{6}^{3} W_{6}^{0} W_{6}^{5} W_{6}^{2} W_{6}^{5} W_{6}^{2} W_{6}^{3} W_{6}^{3} W_{6}^{0} W_{6}^{0} W_{6}^{0} W_{6}^{0} W_{6}^{0} W_{6}^{0} W_{6}^{2} W_{6}^{5} W_{6}^{5} W_{6}^{2}\right), \\
C_{3}= & W_{6}^{3} W_{6}^{0} W_{6}^{0} W_{6}^{3} W_{6}^{3} W_{6}^{3} W_{6}^{3} W_{6}^{3} W_{6}^{2} W_{6}^{2} W_{6}^{5} W_{6}^{5} W_{6}^{0} W_{6}^{3} W_{6}^{0} W_{6}^{3} W_{6}^{3} W_{6}^{0} W_{6}^{0} W_{6}^{3} W_{6}^{5} W_{6}^{5} W_{6}^{5} W_{6}^{5} \\
& \left.W_{6}^{0} W_{6}^{0} W_{6}^{3} W_{6}^{3} W_{6}^{0} W_{6}^{3} W_{6}^{0} W_{6}^{3} W_{6}^{5} W_{6}^{2} W_{6}^{2} W_{6}^{5} W_{6}^{3} W_{6}^{3} W_{6}^{3} W_{6}^{3} W_{6}^{0} W_{6}^{0} W_{6}^{3} W_{6}^{3} W_{6}^{2} W_{6}^{5} W_{6}^{2} W_{6}^{5}\right) .
\end{aligned}
$$


It is worth mentioning that the above set cannot be obtained by using the methods of [4] and [6] and, moreover, although Corollary 4 promises an $\left(L N, N_{r}, L\left(N^{\prime}-1\right)\right)=(48,4,8)$ family, $\mathbf{C}$ is actually a $(48,4,9)$ one. The larger ZCZ is due to the inherit correlation properties of MOCSS

$$
\sum_{k=0}^{N_{r}-1} \psi_{E_{i, k} E_{j,|k \pm 1| N_{r}}}(\tau)=0
$$

for $\tau= \pm(L-1), 0 \leq i<N_{r}$, and $0 \leq j<N_{r}$.

\section{CONCLUSION}

Three new systematic approaches-a transform domain method and two direct (time domain) synthesis methods-for generating ZCZ sequence families have been presented in this paper. The transform domain approach exploits the cross-correlation function's transform domain representation and the recursive Kronecker structure of a class of Hadamard matrices. The two other approaches begin with simple binary basic ZCZ sequences. Through progressively fine-tuning steps that include novel basic sequence-based nonuniform upsampling of unitary matrices or a collections of MOCSS's, we are able to obtain polyphase sequences that meet various ZCZ requirements.

The basic sequences are used to ensure that the required ZCZ width is satisfied during the upsampling process while the transform domain approach uses the subperiodicity of the Hadamard product of two transform domain sequences. The orthogonality among rows of unitary matrices or MOCSS guarantees that the $\mathrm{CC}$ value of any two member sequences at zero lag is zero as well. We take advantage of the correlation-invariant property of the filtering-by-perfectsequence operation to convert a nonconstant modulus sequence into a polyphase sequence. Judicious choices of the basic and perfect sequences used and the associated upsampling rate are crucial in this operation.

Our approaches are conceptually simple and require no sophisticated algebra but, in some cases, offer more flexibilities in either the choices of the sequence length, the ZCZ width and/or the alphabet size needed. We are therefore able to produce sequence families with the same parameters as those by earlier proposals as well as some that are not achievable by related 
known methods. Finally, for each approach, numerical examples have been provided to further validate the proposed construction methods.

\section{APPENDIX A}

\section{PROOFS OF THEOREM 2 AND COROLLARY 4}

Let $P=\left(p_{0}, p_{1}, \cdots, p_{N-1}\right)$ be a row of $\mathbf{P}$ and $C=P \circ A=\left(c_{0}, c_{1}, \cdots, c_{N-1}\right)$, where $c_{n}=\sum_{j=0}^{N-1} p_{j} a_{|j-n|_{N}}^{*}$. If we can show that, for any $n \in[0, N-1]$, one and only one of the $N$ products $\left\{p_{j} a_{|j-n|_{N}}^{*}: j=0, \cdots, N-1\right\}$ is nonzero, then, as both $A$ and $P$ consist 0 's and polyphase elements, $C$ is a polyphase sequence as well. Because of the circular convolution nature of the filtering operation (Definition 4) and the periodic run property of $P$, we have only to check if this single nonzero product assertion is valid for $0<n<N_{r}$.

For the first construction (23), $\operatorname{gcd}\left(N_{r}, N^{\prime}\right)=1$ and both $N_{r}$ and $N^{\prime}$ are positive, hence $\exists$ unique $a, b \in \mathbb{Z}$ such that $a N^{\prime}+b N_{r}=1$, where one of the integer coefficients $a$ or $b$ must be negative [29]. Without loss of generality, we assume $b<0$ and multiply both sides of the above Bézout's identity by $s, 0<s<N_{r}$, to obtain $s a N^{\prime}=s+s b^{\prime} N_{r}, b^{\prime}=-b>0$. If $s b^{\prime} \leq N^{\prime}-1$ then $s a N^{\prime}<N^{\prime} N_{r}=N$ and $s a<N_{r}$; otherwise, subtract both sides by $n_{0} N$, where $n_{0}=\left\lfloor\frac{s b^{\prime} N_{r}}{N}\right\rfloor$ to obtain $\left(s a-n_{0} N_{r}\right) N^{\prime}=s+\left(s b^{\prime}-n_{0} N^{\prime}\right) N_{r}$. For both cases, we have, for each positive $s<N_{r}, \exists$ unique pair of positive integers $(m, n), 0<m \leq N_{r}-1,0 \leq n \leq N^{\prime}-1$ such that $m N^{\prime}=s+n N_{r} \bmod N$. That this property holds for $s=0$ is obvious.

As for the second construction (24), we notice that the basic sequence admits the orthogonal tone decomposition, $B=\sum_{\ell=0}^{d-1} B_{\ell}$, where

$$
B_{\ell}(i)= \begin{cases}b_{i}, & \ell L_{0} \leq i<(\ell+1) L_{0} \\ 0, & \text { otherwise }\end{cases}
$$

When $d=\operatorname{gcd}\left(N_{r}, N^{\prime}\right)$, there exists positive integers $a, b^{\prime}$ such that $a N^{\prime}=d+b^{\prime} N_{r}$. Multiplying both sides by $s, 0 \leq s<\frac{N}{d}$, we obtain $\left(s a-n_{0} \frac{N_{r}}{d}\right) N^{\prime}=s d+\left(s b^{\prime}-n_{0} \frac{N^{\prime}}{d}\right) N_{r}$, where $n_{0}=$ $\left\lfloor\frac{s a N^{\prime}}{L_{0}}\right\rfloor$. For all $s \in\left\{0,1, \cdots, \frac{\left\lfloor N_{r} / N^{\prime}\right\rfloor N^{\prime}+\left(N-N_{r}\right)}{d}\right\}, \exists$ a unique integer pair $(m, n), 0 \leq m<\frac{N_{r}}{d}$, $0 \leq n<N^{\prime}$ such that $m N^{\prime}=s d+n N_{r} \bmod N$, i.e., the sequence $B_{0} \circ A$ is identically zero 
except at indices that are multiples of $d$ and the nonzero terms are the products of two polyphase signals whence are themselves polyphase signals.

Similarly, we can show that, for $\ell=1,2, \cdots, d-1$, the sequence $B_{\ell} \circ A$, has nonzero polyphase terms at $n d-\ell$ only, where $n \in \mathbb{Z}$. Hence the sequence $B \circ A=\sum_{\ell=0}^{d-1} B_{\ell} \circ A$ is a polyphase sequence.

To prove Corollary 4, we first note that the sequences generated differs from those generated by Theorem 2 in that the perfect sequence used in Corollary 4 is the $L$-fold upsampled version of that used in Theorem 2 while the unfiltered $\mathrm{ZCZ}$ sequences for the former is an $L$-expanded version of those for the latter, replacing each zero entry of $P$ by a length- $L$ string of zeros and each nonzero entry by a complementary sequence $E_{i j}$ of length $L$.

For the first construction of $B$ (23), we immediately have, for $0 \leq s<N_{r}, \exists$ unique pair of positive integers $(m, n), 0<m \leq N_{r}-1,0 \leq n \leq N^{\prime}-1$ such that $m L N^{\prime}=s L+n L N_{r}=$ $k+n L N_{r}$. That is, in computing the filtered sequence $C=G \circ A=\left\{c_{k}\right\}$, where $G=\left\{g_{k}\right\} \stackrel{\text { def }}{=} G_{i}$ and $c_{k}=\sum_{j=0}^{L N-1} g_{j} a_{|j-k|_{L N}}^{*}$, there is only one nonzero term in the summands that add up to $c_{k}$, for $k=s L, s=0,1, \cdots, N_{r}-1$. That this single nonzero convolution term property holds for $s L<k<(s+1) L$ is obvious because of the special structure of $G_{i}$. The proof for the case when the second construction (24) is employed follows a similar line of argument.

\section{REFERENCES}

[1] L. R. Welch, "Lower bounds on the maximum cross correlation of signals," IEEE Trans. Inf. Theory, vol. 20, no. 3, pp. 397-399, May 1976.

[2] D. V. Sarwate, "Bounds on cross correlation and autocorrelation of sequences," IEEE Trans. Inf. Theory, vol. 25, no. 6, pp. 720-724, Nov. 1979.

[3] P. Fan, N. Suehiro, N. Kuroyanagi, and X. Deng, "Class of binary sequences with zero correlation zone," Electron. Lett., vol. 35, no. 10, pp. 777-779, May 1999.

[4] X. Deng and P. Fan, "Spreading sequence sets with zero correlation zone," Electron. Lett., vol. 36, no. 11, pp. 993-994, May 2000.

[5] R. Appuswamy and A. K. Chaturvedi, "A new framework for constructing mutually orthogonal complementary sets and ZCZ sequences," IEEE Trans. Inf. Theory, vol. 52, no. 8, pp. 3817-3826, Aug. 2006.

[6] X. Tang, P. Fan, and J. Lindner, "Multiple binary ZCZ sequence sets with good cross-correlation property based on complementary sequence sets," IEEE Trans. Inf. Theory, vol. 56, no. 8, pp. 4038-4045, Aug. 2010. 
[7] H. Torii, M. Nakamura, and N. Suehiro, “A new class of zero-correlation zone sequences," IEEE Trans. Inf. Theory, vol. 50, no. 3, pp. 559-565, Mar. 2004.

[8] X. Tang and W. H. Mow, "A new systematic construction of zero correlation zone sequences based on interleaved perfect sequence," IEEE Trans. Inf. Theory, vol. 54, no. 12, pp. 5729-5734, Dec. 2008.

[9] H. Hu and G. Gong, "New sets of zero or low correlation zone sequences via interleaving techniques," IEEE Trans. Inf. Theory, vol. 56, no. 4, pp. 1702-1713, Apr. 2010.

[10] T. Hayashi, "A novel class of 2-D binary sequences with zero correlation zone," IEEE Signal Process. Lett., vol. 17, no. 3, 301-304, Mar. 2010.

[11] Y. Tu, P. Fan, L. Hao, and X. Li, "Construction of binary array set with zero correlation zone based on interleaving technique," IEICE Trans. Fundamentals, vol. E94-A, no. 2, pp. 766-772, Feb. 2011.

[12] T. Hayashi, "A class of zero-correlation zone sequence set using a perfect sequence," IEEE Signal Process. Lett., vol. 16, no. 4, 331-334, Apr. 2009.

[13] B. M. Popovic and O. Mauritz, "Generalized chirp-like sequences with zero correlation zone," IEEE Trans. Inf. Theory, vol. 56, no. 6, pp. 2957-2960, Jun. 2010.

[14] S. I. Park, S. R. Park, I. Song, and N. Suehiro, "Multiple-access interference reduction for QS-CDMA systems with a novel class of polyphase sequences," IEEE Trans. Inf. Theory, vol. 46, no. 4, pp. 1448-1458, Jul. 2000.

[15] L.-S. Tsai and Y. T. Su, “Transform domain approach for sequence design and its applications," IEEE J. Sel. Areas Commun., vol. 24, no. 1, pp. 75-83, Jan. 2006.

[16] A. K. Brodzik, "New polyphase sequence sets with all-zero cross-correlation," in Proc. IEEE Int. Symp. Inf. Theory, pp. 1421-1424, Boston, MA, USA, Jul. 2012.

[17] H. Torii, T. Matsumoto, and M. Nakamura, "A new method for constructing asymmetric ZCZ sequence sets," IEICE Trans. Fundamentals, vol. E95-A, no. 9, pp. 1577-1586, Sep. 2012.

[18] T. Hayashi, T. Maeda, and S. Matsufuji, "A general construction scheme of a zero-correlation zone sequence set with a wide inter-subset zero-correlation zone," IEICE Trans. Fundamentals, vol. E95-A, no. 11, pp. 1931-1936, Nov. 2012.

[19] F. Zeng, X. Zeng, Z. Zhang, and G. Xuan, "16-QAM sequences with zero correlation zone from known binary ZCZ sequences and Gray mapping," IEICE Trans. Fundamentals, vol. E94-A, no. 11, pp. 2466-2471, Nov. 2011.

[20] Y. Li and C. Xu, "Zero correlation zone sequence set over the 8-QAM+ constellation," IEEE Commun. Lett., vol. 16, no. 11, 1844-1847, Nov. 2012.

[21] B. P. Schweitzer, "Generalized complementary codes," Ph.D. dissertation, Univ. Calif., Los Angeles, 1971.

[22] C.-C. Tseng and C. L. Liu, "Complementary sets of sequences," IEEE Trans. Inf. Theory, vol. 18, no. 5, pp. 644-652, Sep. 1972.

[23] X. H. Tang, P. Fan, and S. Matsufuji, "Lower bounds on correlation of spreading sequence set with low or zero correlation zone," Electron. Lett., vol. 36, no. 6, pp. 551-552, Mar. 2000.

[24] K. J. Horadam, Hadamard Matrices and Their Applications, Princeton, NJ: Princeton University Press, 2007.

[25] C.-W. Chen, Y.-C. Liu, and Y. T. Su, "Systematic constructions of zero-correlation zone sequences," in Proc. IEEE Int. Symp. Inf. Theory, pp. 119-123, Seoul, Korea, Jun. 2009. 
[26] P. Fan and M. Darnell, "The synthesis of perfect sequences," in Proc. IMA Conference on Cryptography and Coding, pp. 63-73, Cirencester, UK, Dec. 1995.

[27] W. H. Mow, "A new unified construction of perfect root-of-unity sequences," in Proc. IEEE Int. Symp. Spread Spectrum Techniques and Applications, pp. 955-959, Mainz, Germany, Sep. 1996.

[28] W. H. Mow, “A study of correlation of sequences," Ph.D. thesis, the Chinese Univ. of Hong Kong, Hong Kong, China, 1993.

[29] J.-P. Tignol, Galois’ Theory of Algebraic Equations, Singapore: World Scientific, 2001.

[30] C.-W. Chen, "On zero-correlation zone sequences,” M.S. thesis, Nat'l Chiao Tung Univ., Hsinchu, Taiwan, 2006. 